\title{
QUASIPLANAR DIAGRAMS AND SLIM SEMIMODULAR LATTICES
}

\author{
GÁBOR CZÉDLI
}

\begin{abstract}
For elements $x$ and $y$ in the (Hasse) diagram $D$ of a finite bounded poset $P, x$ is on the left of $y$, written as $x \lambda y$, if $x$ and $y$ are incomparable and $x$ is on the left of all maximal chains through $y$. Being on the right, written as $x \varrho y$, is defined analogously. The diagram $D$ is quasiplanar if $\lambda$ and $\varrho$ are transitive and for any pair $(x, y)$ of incomparable elements, if $x$ is on the left of some maximal chain through $y$, then $x \lambda y$. A planar diagram is quasiplanar, and $P$ has a quasiplanar diagram iff its order dimension is at most 2 . We are interested in diagrams only up to similarity. A finite lattice is slim if it is join-generated by the union of two chains. The main result gives a bijection between the set of (the similarity classes of) finite quasiplanar diagrams and that of (the similarity classes of) planar diagrams of finite slim semimodular lattices. This bijection allows one to describe finite posets of order dimension at most 2 by finite slim semimodular lattices, and conversely. As a corollary, we obtain that there are exactly $(n-2)$ ! quasiplanar diagrams of size $n$.
\end{abstract}

\section{INTRODUCTION}

1.1. Motivation and aim. Our original goal was to describe finite slim semimodular lattices $L$ by the posets (partially ordered sets) Mi $L=\langle$ Mi $L ; \leq\rangle$ of their meet-irreducible elements. This was motivated by three facts: there are many results on lattices with unique meet irreducible decompositions, slim semimodular lattices have intensively been studied recently, and it is well-known that finite distributive lattices can be described in this way.

Dilworth [21] was the first to deal with unique meet irreducible decompositions in finite lattices. To give a brief overview, let $x^{*}$ denote the join of all covers of $x$ in a finite lattice $L$. If the interval $\left[x, x^{*}\right]$ is distributive for all $x \in L$, then $L$ is a join-distributive lattice in current terminology. There are more than a dozen equivalent definitions of these lattices and two equivalent concepts, antimatroids and convex geometries. Dilworth [21], who was the first to consider these lattices, used the (equivalent) definition that each element can be uniquely decomposed into an irredundant meet of meet irreducible elements. The early variants were surveyed in Monjardet [28]. Since it would wander too far if we overviewed the rest, we only mention Adaricheva [2], Abels [1], Caspard and Monjardet [7], Avann [6], Jamison-Waldner [26], and Ward [32] for additional sources, and Stern [31], Adaricheva and Czédli [3], and Czédli [10] for some recent overviews. However, the

Date: July 10, 2015.

1991 Mathematics Subject Classification. Primary 06C10; secondary 06A06, 06A07.

Key words and phrases. Semimodular lattice, planar lattice, slim lattice, quasiplanar diagram, antimatroid, join-distributive lattice.

This research was supported by the NFSR of Hungary (OTKA), grant numbers K77432 and K83219. 
reader is not assumed to be familiar with these sources, since the present paper is intended to be self-contained for those who know the rudiments of lattice theory up to, say, the Jordan-Hölder Theorem for semimodular lattices. What is mainly important for us is that slim semimodular lattices, to be defined soon, are known to be join-distributive, see Czédli, Ozsvárt, and Udvari [16, Corollary 2.2].
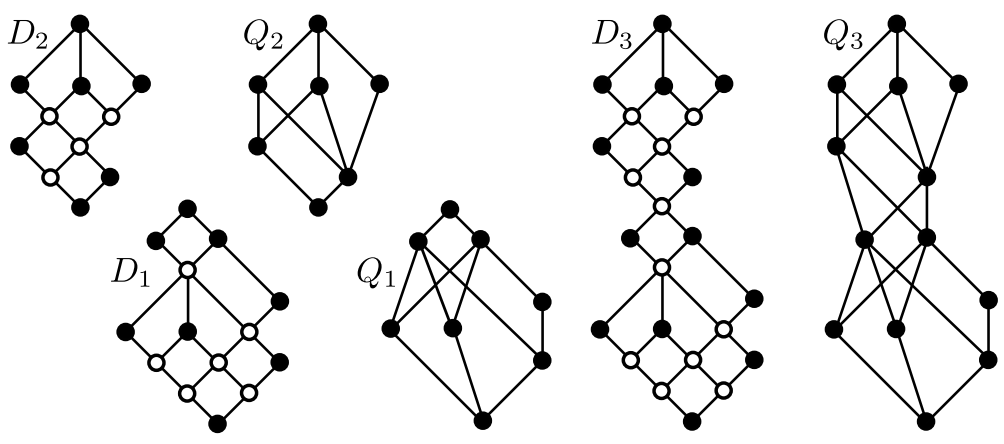

Figure $1 . D_{i}$ and $Q_{i}=\alpha\left(D_{i}\right)$, for $i \in\{1,2,3\}$

A finite lattice $L$ is slim, if Ji $L$, the set of nonzero join-irreducible elements of $L$, is included in the union of two appropriate chains of $L$; see Czédli and Schmidt [17]. For example, it follows trivially from Czédli and Schmidt [18] that the diagrams $D_{1}, \ldots, D_{9}$ and $E^{\prime}$ in Figures 1, 3, 4, 5, and 7 represent slim semimodular lattices. In the semimodular case, the concept of slimness was introduced by Grätzer and Knapp [23] in a slightly different way. The theory of slim semimodular lattices has developed a lot recently, as witnessed by Czédli [8], [9], and [12], Czédli, Dékány, Ozsvárt, Szakács, and Udvari [13], Czédli and Grätzer [14], Czédli, Ozsvárt, and Udvari [16], Czédli and Schmidt [17], [18], [19], and [20], Grätzer and Knapp [23], [24], and [25], and Schmidt [30]. In particular, [17] gives an application of these lattices outside lattice theory while [8], [14], [18], [19], [20], and [23], partly or fully, are devoted to their structural descriptions.

All lattices and posets in the paper are assumed to be finite, even if this convention is not repeated all the time. We have already mentioned that slim semimodular lattices are join-distributive. This fact, combined with Dilworth's original definition of these lattices, and some recent propositions in Czédli [11] led to our original goal, mentioned at the beginning of the paper. Since the poset Mi $L$ does not determine a slim semimodular lattice $L$ in general, the original target had to be modified.

Slim lattice are planar by Czédli and Schmidt [17, Lemma 2.1], that is, they allow planar (Hasse) diagrams. Although the corresponding posets Mi $L$ are not planar in general, their appropriate diagrams still have some important properties of planar ones; we will coin the name quasiplanar for the collection of these properties. For a first impression, note that $Q_{1}, Q_{2}$, and $Q_{3}$ in Figure 1 are quasiplanar diagrams, and they are not planar. The diagrams $Q_{4}, \ldots, Q_{9}$ in Figures 3, 4, and 7 are also quasiplanar, but $Q_{10}$ in Figure 7 is not.

Now, the modified target is to describe the planar diagrams of slim semimodular lattices by quasiplanar diagrams. Of course, diagrams are only considered up to similarity, to be defined soon. The main result of the paper, Theorem 2.11 , gives a canonical bijection between the class of planar diagrams of slim semimodular 
lattices and that of quasiplanar diagrams. For example, for $i \in\{1, \ldots, 9\}$ in our figures, $D_{i}$ corresponds to $Q_{i}$ under this bijection. Having the canonical bijection, even the original goal is achieved in a weak sense, because $L$ is described by any of its planar diagrams $D$, and $D$ is described by a quasiplanar diagram, which is much smaller than $D$ in general. Also, the canonical bijection given by Theorem 2.11 yields a "converse" description, because it describes quasiplanar diagrams by planar diagrams of slim semimodular lattices. This converse description is also interesting, because slim semimodular lattices are well-studied. Its strength will be demonstrated by Corollary 2.12, which counts quasiplanar diagrams of a given size.

1.2. Outline. After recalling or introducing the necessary concepts, Section 2 formulates the main result, Theorem 2.11, which asserts that finite planar slim semimodular lattice diagrams and finite quasiplanar diagrams mutually determine each other. Also, this section gives the exact number of $n$-element quasiplanar diagrams, see Corollary 2.12. Section 3, which contains many auxiliary statements, is devoted to the proof of Theorem 2.11 and that of Corollary 2.12. Finally, Section 4 contains some comments and examples that shed more light on the main result.

1.3. Prerequisites. As mentioned already, the reader is not assumed to have deep knowledge of semimodular lattices; a little part of any book on lattices (or on semimodular lattices), including Grätzer [22], Nation [29], and Stern [31], is sufficient.

\section{Some CONCEPTS AND the MAin RESult}

2.1. Quasiplanar diagrams. The length of a poset $P=\langle P ; \leq\rangle$ is the largest number $n$ such that $P$ has an $(n+1)$-element chain. It will be denoted by length $(P)$. A (Hasse) diagram $D$ of $P$ consists of some points on the plane, representing the elements of $P$, and edges, which are non-horizontal straight line segments connecting two points and represent the covering relation in $P$ in the usual way. Concepts and properties originally defined for posets (and lattices if $P$ happens to be lattice) will also be used for their diagrams; for example, we can speak of a maximal chain or the length of a diagram, and we can say that a lattice diagram is slim and semimodular. A diagram is planar if its edges do not intersect, except possibly at their endpoints. For a more exact definition of planarity and the concepts defined in the next paragraph, the reader can (but need not) resort to Kelly and Rival [27]. Besides planar diagrams, a planar lattice can also have non-planar diagrams. Let us agree that a slim semimodular lattice diagram is always assumed to be planar, even when this is not mentioned.

Let $C$ be a maximal chain in a diagram $D$. This chain cuts $D$ into a left side and a right side, see Kelly and Rival [27, Lemma 1.2]. This is so even if $D$ is not planar but bounded, because $C$ corresponds to a polygon in the plane. The intersection of the left and right sides of $C$ is $C$. If $x \in D$ is on the left side of $C$ but not in $C$, then $x$ is strictly on the left of $C$. The most frequently used results of Kelly and Rival [27] are the following three; note that the second follows easily from the first.

Lemma 2.1 (Kelly and Rival [27, Lemma 1.2]). Let $D$ be a finite planar lattice diagram, and let $x \leq y \in D$. If $x$ and $y$ are on different sides of a maximal chain $C$ in $L$, then there exists an element $z \in C$ such that $x \leq z \leq y$.

Lemma 2.2 (Kelly and Rival [27, Proposition 1.4]). Let $D$ be a planar diagram of a finite lattice $L$. If $C$ is a maximal chain of $D$, then the left side of $C$ and the right side of $C$ "are" (that is, correspond to) sublattices of $L$. 

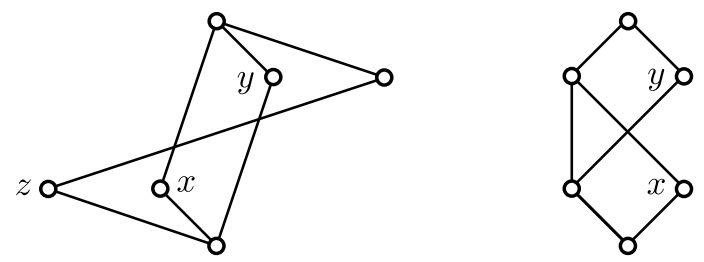

Figure 2. Two diagrams that are not quasiplanar

Lemma 2.3 (Kelly and Rival [27, Proposition 1.6]). Let $D$ be a finite planar lattice diagram, and let $x, y \in L$ be incomparable elements. If $x$ is on the left of some maximal chain (of $D$ ) through $y$, then $x$ is on the left of every maximal chain through $y$.

Next, we turn our attention to diagrams of posets. We will only consider bounded diagrams, that is diagrams with 0 and 1 , because otherwise the meaning of the left or right side of a maximal chain, which is possibly a singleton, is less pictorial. As usual, \| stands for the incomparability relation; $x \| y$ means that $x \not \leq y$ and $y \not \leq x$. For $x \| y$ in the diagram of a poset, we say that $x$ is on the left of $y$, written as $x \lambda y$ (resp., $x$ is on the right of $y$, written as $x \varrho y$ ) if $x$ is on the left (resp., right) of all maximal chains through $y$. Let us agree and emphasize that $x \lambda y \Rightarrow x \| y$ and $x \varrho y \Rightarrow x \| y$. The following definition is motivated by Lemma 2.3 and by further properties that are stated for planar lattice diagrams in Kelly and Rival [27]. However, in general, Lemma 2.1 will not be valid for quasiplanar diagrams, which play a crucial role in the paper.

Definition 2.4. A diagram $D$ of a finite poset is quasiplanar if it is bounded and it satisfies the following three axioms for all $x, y, z \in D$.

(A1) If $x \| y$ and $x$ is on the left of some maximal chain through $y$, then $x$ is on the left of all maximal chains through $y$, that is, then $x \lambda y$.

(A2) If $x \lambda y$ and $y \lambda z$, then $x \lambda z$.

(A3) If $x \varrho y$ and $y \varrho z$, then $x \varrho z$.

Let us emphasize that, by definition, a quasiplanar diagram in the present paper is always finite and has 0 and 1 . (The more general concept of diagrams that can be extended to quasiplanar diagrams by adding a bottom element and a top element will not be used.) The first diagram in Figure 2 indicates that (A1) does not imply (A2). The second diagram in the figure shows that (A1) and (A2) together do not imply (A3), since (A1) and (A2) hold, $x \varrho y$, and $y \varrho x$, but not $x \varrho x$. Note that both diagrams in the figure determine planar lattices, that is, lattices that also have planar diagrams. Some important properties of quasiplanar diagrams are revealed by the following lemma. To point out a less important but interesting fact, consider $Q_{8}$ in Figure 4, and let $Q_{8}^{-}$be the diagram that we obtain from $Q_{8}$ by deleting the edge $b \prec e$. (That is, $Q_{8}^{-}$is the usual diagram of the 8 -element boolean lattice.) While $Q_{8}$ is quasiplanar, $Q_{8}^{-}$is not.

Lemma 2.5. A quasiplanar diagram $D$ satisfies the following four properties for all $x, y, z \in D$.

(A4) If $x \| y$ and $x$ is on the right of some maximal chain through $y$, then $x \varrho y$.

(A5) If $x \| y$, then exactly one of $x \lambda y$ and $y \lambda x$ holds. 
(A6) $x \lambda y \Longleftrightarrow y \varrho x$.

(A7) If $x \lambda y$ and $y \nVdash z$, then either $x \lambda z$, or $x \nVdash z$.

Proof. Assume that there exist maximal chains $C_{1}$ and $C_{2}$ through $y$ such that $x$ is on the left of $C_{1}$ and on the right of $C_{2}$. It follows from (A1) that $x$ is also on the left of $C_{2}$. Hence, $x \in C_{2}$, which contradicts $x \| y$. Thus, (A4) holds in $D$.

Next, assume $x \| y$. If $x \lambda y$ and $y \lambda x$ both hold, then $x \lambda x$ by (A2), which contradicts $x \nVdash x$. Suppose that neither $x \lambda y$ nor $y \lambda x$ holds. Take a maximal chain $C$ through $y$. Since $x \lambda y$ is excluded, $x$ is on the right of $C$, and we have $x \varrho y$ by (A4). Similarly, $y \varrho x$. Using (A3), we obtain $x \varrho x$, which contradicts $x \nVdash x$. Hence, $D$ satisfies (A5) .

Next, suppose that $x \lambda y$ holds but $y \varrho x$ fails. Since the role of left and right is symmetric in the collection of (A1), .., (A4), the symmetric counterpart of (A5) holds, and it implies $x \varrho y$. If $C$ is a maximal chain through $y$, then $x$ is on the left of $C$ by $x \lambda y, x$ is on the right of $C$ by $x \varrho y$, and $x \notin C$ by $x \| y$. This contradiction proves $x \lambda y \Rightarrow y \varrho x$, while the converse implication follows by left-right symmetry. Hence, (A6) holds in $D$.

Finally, to prove (A7), assume $x \lambda y$ and $y \nVdash z$. Suppose, for a contradiction, that neither $x \lambda z$, nor $x \nVdash z$. Combining (A5) and (A6), we obtain $x \varrho z$. Let $C$ be a maximal chain through $\{y, z\}$. Since $x \lambda y, x$ is on the left of $C$. On the other hand, $x \varrho z$ yields that $x$ is on the right of $C$. Hence, $x \in C$, which contradicts $x \| y$.

Combining Kelly and Rival [27, Proposition 1.7 and Corollary 2.4] with Lemma 2.3 and using that the role of left and right is symmetric, we obtain the following statement.

Lemma 2.6. If $D$ is a planar diagram of a finite bounded poset $P$, then $D$ is a quasiplanar diagram and $P$ is a lattice.

If $D^{\prime}$ and $D^{\prime \prime}$ are quasiplanar diagrams and there exists a bijection $\psi: D^{\prime} \rightarrow D^{\prime \prime}$ such that $\psi$ is an order isomorphism and, for any $x, y \in D^{\prime}, x \lambda y$ in $D^{\prime}$ iff $\psi(x) \lambda \psi(y)$ in $D^{\prime \prime}$, then $D^{\prime}$ and $D^{\prime \prime}$ are similar diagrams and $\psi$ is a similarity map. In this way, as it follows from Lemma 2.6, we have also defined the concept of similarity for planar lattice diagrams. Note that for planar lattice diagrams, similarity means the same as in Kelly and Rival [27]. We consider quasiplanar diagrams and planar lattice diagrams up to similarity; that is, similar diagrams will always be treated as equal ones, even if this is not repeated all the time.

For a planar lattice diagram $D$, let $C$ and $E$ be maximal chains of $D$. If all elements of $E$ are on the left of $C$, then $E$ is on the left of $C$. In this sense, we can speak of the leftmost maximal chain of $D$, called the left boundary chain, and the rightmost maximal chain, called the right boundary chain. The union of these two chains is the boundary of $D$. The assumption that $D$ is a planar lattice diagram is important in this paragraph, because, say, the second quasiplanar diagram in Figure 2 does not have a right boundary chain. If $F$ is a (not necessarily maximal) chain of the planar lattice diagram $D$, then the leftmost maximal chain through $F$ (or extending $F$ ) and the rightmost one make sense. If $F=\left\{f_{1}<\cdots<f_{n}\right\}$, then the leftmost maximal chain of $D$ through $F$ is the union of the left boundary chains of the subdiagrams $\downarrow f_{1}=\left\{x \in D: x \leq f_{1}\right\},\left[f_{1}, f_{2}\right], \ldots,\left[f_{n-1}, f_{n}\right]$, and $\uparrow f_{n}=\left\{x \in D: x \geq f_{n}\right\}$. If $F=\{f\}$ is a singleton, then chains containing $f$ are said to be chains through $f$ rather than chains through $\{f\}$. 
An important tool to recognize similarity is given in the following lemma, which is taken from Czédli and Schmidt and [20, Lemma 4.7] or, more explicitly, Czédli and Grätzer [15].

Lemma 2.7. Let $D^{\prime}$ and $D^{\prime \prime}$ be planar slim semimodular lattice diagrams. If there exists an order-isomorphism $\psi: D^{\prime} \rightarrow D^{\prime \prime}$ such that $\psi$ maps the left boundary chain of $D^{\prime}$ to the left boundary chain of $D^{\prime \prime}$, then $D^{\prime}$ and $D^{\prime \prime}$ are similar diagrams and $\psi$ is a similarity map.
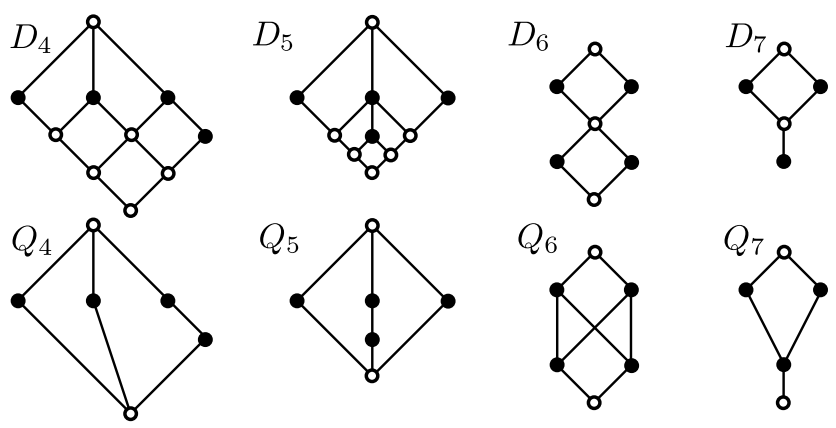

FiguRE 3. $D_{i}$ and $Q_{i}=\alpha\left(D_{i}\right)$, for $i \in\{4,5,6,7\}$

2.2. The key constructions. Before formulating the main result, we have to give the basic constructions. It is not so trivial that our constructs exist and have the desired properties, but this will be proved later, in due time. The following definition is illustrated by Figures 1,3 , and 4 , and also by $Q_{9}=\alpha\left(D_{9}\right)$ in Figure 7 . For $i \in\{1, \ldots, 9\}$ in these figures, Mi $D_{i}$ consists of the black-filled elements of $D_{i}$.

Definition 2.8. Let $D$ and $Q$ be a planar lattice diagram and a quasiplanar diagram, respectively. We say that $Q$ is the quasiplanar diagram associated with $D$ if the following three conditions hold.

(i) $Q=\{1, \tilde{0}\} \cup \operatorname{Mi} D$, where $1 \in D, \tilde{0} \notin D$. (The equality here does not mean the equality of points in the plane, since we only consider $Q$ up to similarity.)

(ii) For $x, y \in Q, x \leq y$ in $Q$ iff $x \leq y$ in $D$ or $x=\tilde{0}$.

(iii) For any two incomparable $x, y \in Q$, we have $x \lambda y$ in $Q$ iff $x \lambda y$ in $D$.

If $Q$ above exists, then it is clearly unique up to similarity; it is denoted by $\alpha(D)$.

We do not claim that $Q$ above exists for every $D$. Since $\lambda=\lambda_{Q}$ is the relation "on the left" on $Q, x \lambda^{=} y$ will mean that either $x=y$ or $x \lambda y$. We define the relations $\lambda^{\leq}, \lambda^{\geq}, \lambda^{<}, \lambda^{>}, \varrho^{=}, \varrho^{\leq}, \varrho^{\geq}, \varrho^{<}$, and $\varrho^{>}$analogously; for example, $x \lambda^{\leq} y$ means that $x \leq y$ or $x \lambda y$, and $x \varrho^{>} y$ means $x>y$ or $x \varrho y$.

Next, we start from a quasiplanar diagram, and want to define a planar slim semimodular lattice diagram; see Figure 4 for an illustration.

Definition 2.9. For a quasiplanar diagram $Q$, let $Q^{+}=Q \backslash\{0\}$, and let $E(Q)$ denote the relation $\lambda^{=}$restricted to $Q^{+}$. That is,

$$
E(Q)=\left\{\langle x, y\rangle \in Q^{+} \times Q^{+}: x \lambda^{=} y\right\} .
$$

For $\left\langle x_{1}, y_{1}\right\rangle,\left\langle x_{2}, y_{2}\right\rangle \in E(Q)$, we define

$$
\left\langle x_{1}, y_{1}\right\rangle \leq\left\langle x_{2}, y_{2}\right\rangle \stackrel{\text { def }}{\Longleftrightarrow} x_{1} \lambda^{\leq} x_{2} \text { and } y_{2} \lambda^{\geq} y_{1} \text {, and }
$$



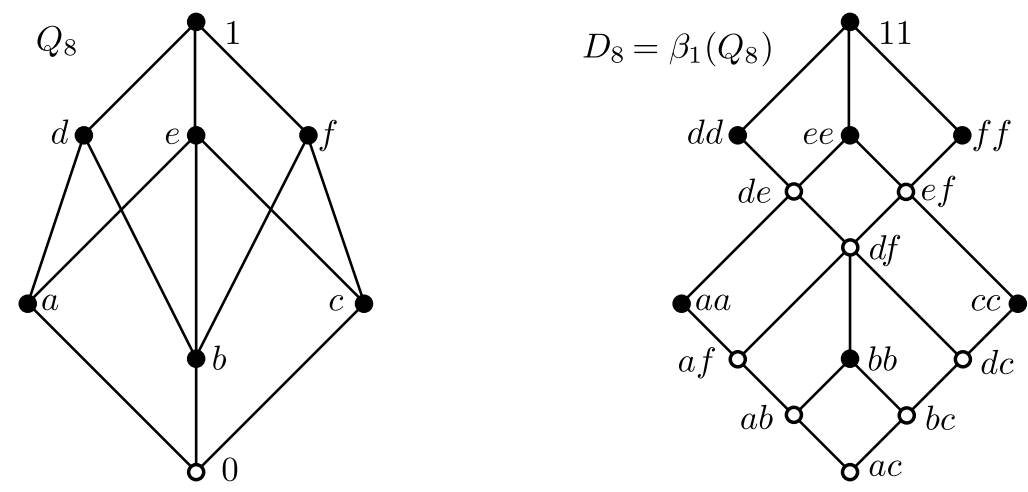

Figure 4. A quasiplanar diagram $Q_{8}$ and $\beta_{1}\left(Q_{8}\right)$

$$
\left\langle x_{1}, y_{1}\right\rangle \lambda\left\langle x_{2}, y_{2}\right\rangle \stackrel{\text { def }}{\Longleftrightarrow} x_{1} \lambda^{<} x_{2} \text { and } y_{1} \lambda^{>} y_{2} .
$$

(Note that $y_{2} \lambda^{\geq} y_{1}$ in (2.1) is equivalent to $y_{1} \varrho^{\leq} y_{2}$.) Let $\beta_{1}(Q)$ be the unique planar diagram of $\langle E(Q)$; $\leq\rangle$, where " $\leq$ " is given by $(2.1)$, such that the "on the left" relation of $\beta_{1}(Q)$ is described by (2.2). (We will prove that such a diagram exists; its uniqueness is obvious.) The construction is illustrated in Figure 4, where $\langle x, y\rangle$ is written as $x y$. Writing $0 \in Q_{8}$ for $\tilde{0}$, observe that $\alpha\left(D_{8}\right)=Q_{8}$.

The advantage of Definition 2.9 is that the pairs in $E(Q)$ are relatively simple objects and $\lambda$ in $\beta_{1}(Q)$ is quite explicitly described. However, we will also benefit from the following approach in our proofs.

Definition 2.10. Let $Q$ be a quasiplanar diagram, and let $Q^{+}=Q \backslash\{0\}$.

(i) A nonempty subset $X$ of $Q^{+}$is called a proper horizontally convex order filter, in short a hco-filter, of $Q$ if

- $X$ is an up-set, that is, $x \in X, y \in Q$, and $x \leq y$ imply $y \in X$, and

- $X$ is horizontally convex, that is, if $x \lambda y, y \lambda z$, and $\{x, z\} \subseteq X$, then $y \in X$.

(ii) For $Y \subseteq Q^{+}$, the least hco-filter including $Y$ is denoted by $\uparrow^{\text {hco }} Y=\uparrow_{Q}^{\text {hco }} Y$; we write $\uparrow^{\text {hco }} y$ instead of $\uparrow^{\text {hco }}\{y\}$.

(iii) The set of hco-filters of $Q$ is denoted by $F_{\text {hco }}(Q)$. For $X, Y \in F_{\text {hco }}(Q)$, let $X \leq^{d} Y$ mean $X \supseteq Y$; the poset $\left\langle F_{\text {hco }}(Q) ; \leq^{d}\right\rangle$ is also denoted by $F_{\text {hco }}(Q)$.

(iv) We define a finite sequence of hco-filters $\vec{F}(Q)=\vec{F}=\left(F_{0}, F_{1}, \ldots, F_{|Q|-2}\right)$ by induction as follows. Let $F_{0}=\{1\}$. If $F_{n}$ is defined and $Q^{+} \backslash F_{n} \neq \varnothing$, then let $f_{n}$ be the leftmost element in the set $\operatorname{Max}\left(Q \backslash F_{n}\right)$ of maximal elements of $Q \backslash F_{n}$, and let $F_{n+1}=F_{n} \cup\left\{f_{n}\right\}$.

(v) We also define the "left-right dual" version $\vec{G}(Q)=\vec{G}=\left(G_{0}, G_{1}, \ldots, G_{|Q|-2}\right)$ of $\vec{F}$ by induction as follows. Let $G_{0}=\{1\}$. While $Q^{+} \backslash G_{n} \neq \varnothing$, denote by $g_{n}$ the rightmost element of $\operatorname{Max}\left(Q \backslash G_{n}\right)$, and let $G_{n+1}=G_{n} \cup\left\{g_{n}\right\}$.

(vi) Let $\beta_{2}(Q)$ be the planar lattice diagram of $\left\langle F_{\text {hco }}(Q) ; \leq^{d}\right\rangle$ such that $\vec{F}$ and $\vec{G}$ are the left boundary chain and the right boundary chain, respectively. (We will show later that this makes sense.)

2.3. The results. In order to take Definitions 2.9 and 2.10 into account independently, the main theorem below contains a parameter $p \in\{1,2\}$. 
Theorem 2.11 (Main Theorem). Let $D$ be a finite planar slim semimodular lattice diagram, and let $Q$ be a finite quasiplanar diagram. Let $p \in\{1,2\}$. Then the following hold.

(i) $\alpha(D)$ is a finite quasiplanar diagram.

(ii) $\beta_{p}(Q)$ is a finite planar slim semimodular lattice diagram.

(iii) Up to similarity, $\beta_{p}(\alpha(D))$ equals $D$.

(iv) Up to similarity, $\alpha\left(\beta_{p}(Q)\right)$ equals $Q$.

As an application, we will prove the following corollary, which is of separate interest. Let us emphasize that quasiplanar diagrams are bounded by definition.

Corollary 2.12. Up to similarity, the number of n-element quasiplanar diagrams is $(n-2)$ !.

Next, we give an example. For a quasiplanar diagram $D$, let $\operatorname{VFlip}(D)$ denote the vertical mirror image of $D$ across a vertical axis. Clearly, $\operatorname{VFlip}(D)$ is also quasiplanar. If $D$ is the same as $\operatorname{VFlip}(D)$ up to similarity, then $D$ is a left-right symmetric diagram. Armed with this notation and concept, it is easy to list all the $24=(6-2)$ ! quasiplanar diagrams of size 6 (up to similarity) as follows. Let $L_{1}, \ldots, L_{17}$ be the lattices given in Figures 2 and 3 of Czédli, Ozsvárt, and Udvari [16], and let $\widehat{L}_{1}, \ldots, \widehat{L}_{17}$ denote their planar diagrams according to these figures. By [16], $L_{1}, \ldots, L_{17}$ is a (repetition free) complete list of slim semimodular lattices of length 4 , up to isomorphism. Since similar diagrams define isomorphic lattices, the corresponding planar diagrams, $\widehat{L}_{1}, \ldots, \widehat{L}_{17}$, are pairwise non-similar. By reflecting those that are not left-right symmetric, we define $\widehat{L}_{18}:=\operatorname{VFlip}\left(\widehat{L}_{1}\right), \widehat{L}_{19}:=\operatorname{VFlip}\left(\widehat{L}_{4}\right)$, $\widehat{L}_{20}:=\operatorname{VFlip}\left(\widehat{L}_{5}\right), \widehat{L}_{21}:=\operatorname{VFlip}\left(\widehat{L}_{6}\right), \widehat{L}_{22}:=\operatorname{VFlip}\left(\widehat{L}_{8}\right), \widehat{L}_{23}:=\operatorname{VFlip}\left(\widehat{L}_{11}\right)$, and $\widehat{L}_{24}:=\operatorname{VFlip}\left(\widehat{L}_{12}\right)$. It is easy to see that $\widehat{L}_{1}, \ldots, \widehat{L}_{24}$ are pairwise non-similar diagrams. Using Lemma 3.7, see later, and the fact that $L_{1}, \ldots, L_{17}$ is a complete list, it also follows that $\widehat{L}_{1}, \ldots, \widehat{L}_{24}$ is a complete list of slim semimodular lattice diagrams of length 4 up to similarity. (This will also follow from Theorem 2.11 and Corollary 2.12.) By Definition 2.8, it is quite easy to construct the quasiplanar diagrams $\alpha\left(\widehat{L}_{1}\right), \ldots, \alpha\left(\widehat{L}_{24}\right)$. For example, with reference to Figure 3 of the present paper, $\alpha\left(\widehat{L}_{3}\right)=\alpha\left(D_{5}\right)=Q_{5}, \alpha\left(\widehat{L}_{13}\right)=\alpha\left(D_{6}\right)=Q_{6}$, and $\alpha\left(\widehat{L}_{21}\right)=\alpha\left(D_{4}\right)=Q_{4}$. Now, by Theorem 2.11, $\alpha\left(\widehat{L}_{1}\right), \ldots, \alpha\left(\widehat{L}_{24}\right)$ is a complete list of quasiplanar poset diagrams of size 6 . It turns out that all 24 diagrams in the list, except for $\alpha\left(\widehat{L}_{13}\right)=Q_{6}$, are planar lattice diagrams.

\section{Auxiliary statements and proofs}

3.1. Statements on quasiplanar diagrams. Let $Q$ be a quasiplanar diagram, and let $F \in F_{\text {hco }}(Q)$ be a hco-filter. The set of minimal elements of $F$ is denoted by $\operatorname{Min} F$. It is an antichain, so it has a unique leftmost element lbe $(F)$, and a unique rightmost element $\operatorname{rbe}(F)$. They are the leftmost bottom element and the rightmost bottom element of $F$, respectively. Clearly, $\operatorname{lbe}(F) \lambda^{=} \operatorname{rbe}(F)$. If $\langle x, y\rangle \in E(Q)$, then we often use the following notation

$$
\begin{aligned}
\operatorname{Betw}(x, y) & =\left\{z: x \lambda^{=} z \text { and } z \lambda^{=} y\right\} \text { and } \\
\operatorname{Min} \operatorname{Betw}(x, y) & =\operatorname{Min}\left\{z: x \lambda^{=} z \text { and } z \lambda^{=} y\right\},
\end{aligned}
$$


where for an $A \subseteq Q, \operatorname{Min} A$ denotes the set of minimal elements of $A$. Since $x \lambda^{=} y$, the set $\operatorname{Min} \operatorname{Betw}(x, y)$ is not empty. For $U \subseteq Q, \uparrow U$ denotes the order filter $\{z \in Q: z \geq u$ holds for some $u \in U\}$ generated by $U$.

Lemma 3.1. If $Q$ is a quasiplanar diagram, then for any $\langle x, y\rangle \in E(Q)$, we have

(i) $\uparrow^{\text {hco }}\{x, y\}=\uparrow \operatorname{Min} \operatorname{Betw}(x, y)$ and, in particular, $\uparrow^{\text {hco }} x=\uparrow x$;

(ii) $x=\operatorname{lbe}(\uparrow \mathrm{hco}\{x, y\})$ and $y=\operatorname{rbe}\left(\uparrow^{\mathrm{hco}}\{x, y\}\right)$;

(iii) $\operatorname{Min}\left(\uparrow^{\text {hco }}\{x, y\}\right)=\operatorname{Min} \operatorname{Betw}(x, y)$.

Proof. The " $\supseteq$ " inclusion in the first equation of (i) is obvious. Assume that $u_{1}, u_{2} \in \uparrow \operatorname{Min} \operatorname{Betw}(x, y), u \in Q$, and $u_{1} \lambda u \lambda u_{2}$. We want to show $u \in$ $\uparrow \operatorname{Min} \operatorname{Betw}(x, y)$. There are $v_{1}, v_{2} \in \operatorname{Min} \operatorname{Betw}(x, y)$ such that $v_{1} \leq u_{1}$ and $v_{2} \leq u_{2}$. By (A7), either $u \lambda v_{2}$ or $u \nmid v_{2}$. Now $u \leq v_{2}$ would give $u \leq u_{2}$, which would contradict $u \lambda u_{2}$. If we had $u \geq v_{2}$, then $u \in \uparrow \operatorname{Min} \operatorname{Betw}(x, y)$ would trivially hold. Hence we can assume $u \lambda v_{2}$. Similarly, we can also assume $v_{1} \lambda u$. We know that $x \lambda v_{1}$ and $v_{2} \lambda y$. Armed with the formulas $x \lambda v_{1}, v_{1} \lambda u, u \lambda v_{2}$, and $v_{2} \lambda y$, (A2) yields $u \in \operatorname{Betw}(x, y) \subseteq \uparrow \operatorname{Min} \operatorname{Betw}(x, y)$. Therefore, $\uparrow \operatorname{Min} \operatorname{Betw}(x, y)$ is a hco-filter. Finally, it is trivial that $x$ and $y$ belong to $\left\{z: x \lambda^{=} z\right.$ and $\left.z \lambda^{=} y\right\}$, and they are minimal elements in this set. That is, $\{x, y\} \subseteq \operatorname{Min} \operatorname{Betw}(x, y)$, and the " $\subseteq$ " inclusion in (i) follows. This proves the first equation of (i); the second one is a particular case since $\langle x, x\rangle \in E(Q)$.

Obviously, if $A$ is an antichain, then $\operatorname{Min}(\uparrow A)=A$. Applying this fact to $A=$ $\operatorname{Min} \operatorname{Betw}(x, y)$ and taking (i) into account, we conclude (ii) and (iii).

The following lemma says that Definitions 2.9 and 2.10 are quite close to each other.

Lemma 3.2. Given a quasiplanar diagram $Q$, the maps

$$
\varphi: E(Q) \rightarrow F_{\text {hco }}(Q), \quad \text { defined by }\langle x, y\rangle \mapsto \uparrow^{\text {hco }}\{x, y\},
$$

and

$$
\pi: F_{\text {hco }}(Q) \rightarrow E(Q), \quad \text { defined by } F \mapsto\langle\operatorname{lbe}(F), \operatorname{rbe}(F)\rangle,
$$

are reciprocal order isomorphisms.

Proof. Assume that $\left\langle x_{1}, y_{1}\right\rangle \leq\left\langle x_{2}, y_{2}\right\rangle$ in $E(Q)$. This means that $x_{1} \lambda \leq x_{2}$ and $y_{2} \lambda^{\geq} y_{1}$. Let $F_{i}=\uparrow^{\text {hco }}\left\{x_{i}, y_{i}\right\}=\varphi\left(\left\langle x_{i}, y_{i}\right\rangle\right)$ for $i \in\{1,2\}$. To obtain $F_{1} \leq^{d} F_{2}$, that is $F_{2} \subseteq F_{1}$, we have to show $x_{2}, y_{2} \in \uparrow^{\text {hco }}\left\{x_{1}, y_{1}\right\}$. We can assume $x_{1} \not x_{2}$ since otherwise $x_{2} \in \uparrow^{\text {hco }}\left\{x_{1}, y_{1}\right\}$ trivially holds. Thus $x_{1} \lambda x_{2}$. If $y_{2} \lambda y_{1}$, then $x_{1} \lambda x_{2} \lambda^{=} y_{2} \lambda y_{1}$, together with the horizontal convexity of $F_{1}$, yields $x_{2} \in F_{1}$. If $y_{2} \geq y_{1}$, then $y_{2} \in F_{1}, x_{1} \lambda x_{2} \lambda^{=} y_{2}$, and the horizontal convexity of $F_{1}$ yield $x_{2} \in F_{1}$ again. Hence, $x_{2} \in F_{1}$, and $y_{2} \in F_{1}$ follows by left-right duality. Therefore, $\varphi$ is order-preserving.

We know from Lemma 3.1(ii) that $\pi \circ \varphi: E(Q) \rightarrow E(Q)$ is the identity map on $E(Q)$. To prove that $\varphi \circ \pi$ is the identity map on $F_{\text {hco }}(Q)$, let $F \in F_{\text {hco }}(Q)$. Denoting lbe $(F)$ and $\operatorname{rbe}(F)$ by $x$ and $y$, respectively, we have $\pi(F)=\langle x, y\rangle$. We also have $(\varphi \circ \pi)(F)=\varphi(\pi(F))=\uparrow^{\text {hco }}\{x, y\}$. The inclusion $(\varphi \circ \pi)(F)=\uparrow^{\text {hco }}\{x, y\} \subseteq F$ is trivial. To show the converse inclusion, let $u \in F$. Then there exists a $v$ in the antichain Min $F$ such that $u \geq v$. By the definition of $x$ and $y$, we have $x \lambda^{=} v \lambda^{=} y$. Hence $v \in \uparrow^{\text {hco }}\{x, y\}$, which implies $u \in \uparrow^{\text {hco }}\{x, y\}$. This proves that $\varphi \circ \pi$ is the identity map on $F_{\text {hco }}(Q)$, and thus $\varphi$ and $\pi$ are reciprocal bijections. 
Finally, to prove that $\pi$ is order-preserving, assume that $F_{1} \leq^{d} F_{2} \in F_{\text {hco }}(Q)$. Denoting $\pi\left(F_{i}\right)$ by $\left\langle x_{i}, y_{i}\right\rangle$ and using $\pi^{-1}=\varphi$, this gives $\uparrow^{\text {hco }}\left\{x_{1}, y_{1}\right\} \supseteq \uparrow^{\text {hco }}\left\{x_{2}, y_{2}\right\}$. Hence, by Lemma 3.1(i), $\left\{x_{2}, y_{2}\right\} \subseteq \uparrow \operatorname{Min} \operatorname{Betw}\left(x_{1}, y_{1}\right)$. If $x_{2} \geq x_{1}$, then $x_{1} \lambda \leq x_{2}$ is clear. Hence, we assume $x_{2} \geq x_{1}$. It follows trivially or from Lemma 3.1(iii) that $x_{1}$ belongs to the set $\operatorname{Min}\left(\uparrow \operatorname{Min} \operatorname{Betw}\left(x_{1}, y_{1}\right)\right)$, whence $x_{2} \nless x_{1}$. Thus $x_{2} \| x_{1}$. Since $x_{2} \in \uparrow \operatorname{Min} \operatorname{Betw}\left(x_{1}, y_{1}\right)$, there exists a $u \in \operatorname{Min} \operatorname{Betw}\left(x_{1}, y_{1}\right)$ such that $x_{2} \geq u$, and we obtain $x_{1} \lambda x_{2}$ from (A7). Hence, in all cases, $x_{1} \lambda \leq x_{2}$. By left-right duality, we obtain $y_{2} \lambda^{\geq} y_{1}$. Therefore, $\pi\left(F_{1}\right)=\left\langle x_{1}, y_{1}\right\rangle \leq\left\langle x_{2}, y_{2}\right\rangle=\pi\left(F_{2}\right)$.

The concept of antimatroids is due to Jamison-Waldner [26]. We cite the following definition from Armstrong [5, Lemma 2.1].

Definition 3.3. A pair $\langle E, \mathfrak{F}\rangle$ is an antimatroid if it satisfies the following properties:

(i) $E$ is a finite set, and $\mathfrak{F}$ is a nonempty family of subsets of $E$.

(ii) $\mathfrak{F}$ is a feasible set, that is, for each nonempty $A \in \mathfrak{F}$, there exists an $x \in A$ such that $A \backslash\{x\} \in \mathfrak{F}$;

(iii) $\mathfrak{F}$ is closed under taking unions;

(iv) $E=\bigcup\{A: A \in \mathfrak{F}\}$.

The relevance of this concept here is explained by the following well-known statement; see Armstrong [5, Theorem 2.8], who attributes it to Birkhoff, Whitney and MacLane, or Adaricheva, Gorbunov, and Tumanov [4], see also Czédli [10].

Lemma 3.4. If $\langle E, \mathfrak{F}\rangle$ is an antimatroid, then $\langle\mathfrak{F} ; \subseteq\rangle$ is a finite join-distributive lattice. Up to isomorphism, each join-distributive lattice can be obtained this way.

Lemma 3.5. If $Q$ is a quasiplanar diagram, then $\left\langle F_{\mathrm{hco}}(Q) ; \leq^{d}\right\rangle$ is a semimodular lattice.

Proof. Let $\mathfrak{F}=\left\{Q^{+} \backslash F: F \in F_{\text {hco }}(Q)\right\}$ and $E=Q \backslash\{0,1\}$. Then $\mathfrak{F}$ is a family of subsets of $E$ and $\left\langle F_{\text {hco }}(Q) ; \leq^{d}\right\rangle \cong\langle\mathfrak{F} ; \subseteq\rangle$. Since $F_{\text {hco }}(Q)$ is clearly closed with respect to intersections, $\mathfrak{F}$ is closed under taking unions. We claim that $\langle E ; \mathfrak{F}\rangle$ is an antimatroid. This will prove Lemma 3.5, because then Lemma 3.4 applies and joindistributive lattices are semimodular; see, for example, Monjardet [28], JamisonWaldner [26], and see [4], [5], and [10] mentioned a few lines above. Since $E$ and $\varnothing$ belong to $\mathfrak{F}$ and $\mathfrak{F}$ is closed under taking unions, we only have to show that $\mathfrak{F}$ is a feasible set. By the definition of $\mathfrak{F}$, is suffices to prove that if $Q^{+} \neq F \in F_{\text {hco }}(Q)$, then there exists an element $u$ in $Q^{+} \backslash F$ such that $F \cup\{u\} \in F_{\text {hco }}(Q)$. To show this, take a minimal $G \in F_{\text {hco }}(Q)$, with respect to " $\subseteq$ ", such that $F \subset G$; it is sufficient to prove that $|G \backslash F|=1$. By Lemma $3.2, F$ is of the form $\uparrow^{\text {hco }}\{x, y\}$ for some $\langle x, y\rangle \in E(Q)$. There are three cases to discuss, but first we formulate the following three rules.

$$
\begin{gathered}
\left(\forall x_{1}, x_{2}, x_{3} \in Q^{+}\right)(\exists i \in\{1,2,3\})\left(x_{i} \in \uparrow^{\mathrm{hco}}\left(\left\{x_{1}, x_{2}, x_{3}\right\} \backslash\left\{x_{i}\right\}\right)\right) ; \\
\left(\left\langle x_{1}, x_{2}\right\rangle \in E(Q), \text { and } x_{3}<x_{1} \text { or } x_{3}<x_{2}\right) \Rightarrow x_{3} \notin \uparrow{ }^{\mathrm{hco}}\left\{x_{1}, x_{2}\right\} ; \\
x_{1} \lambda x_{2} \lambda x_{3} \Rightarrow\left(x_{1} \notin \uparrow^{\mathrm{hco}}\left\{x_{2}, x_{3}\right\} \text { and } x_{3} \notin \uparrow \uparrow^{\mathrm{hco}}\left\{x_{1}, x_{2}\right\}\right) .
\end{gathered}
$$

The validity of (3.1) is obvious if $\left\{x_{1}, x_{2}, x_{3}\right\}$ is not a three-element antichain, and it follows from the fact that one of the three elements is horizontally between the other two otherwise. To prove (3.2) by way of contradiction, suppose that (3.2) fails. By left-right symmetry, we also assume $x_{3}<x_{1}$. Then $x_{1} \lambda x_{2}$, and 
Lemma 3.1(i) yields a $t$ such that $x_{1} \lambda^{=} t \lambda^{=} x_{2}$ and $t \leq x_{3}$. Hence $x_{1}>x_{3} \geq t$ contradicts $x_{1} \lambda^{=} t$, proving (3.2). Next, it suffices to prove (3.3) only for $x_{1}$, because then the $x_{3}$-part follows by left-right symmetry. By way of contradiction, suppose $x_{1} \lambda x_{2} \lambda x_{3}$ but $x_{1} \in \uparrow^{\text {hco }}\left\{x_{2}, x_{3}\right\}$. By Lemma 3.1(i), there exists a $t$ such that $x_{2} \lambda^{=} t \lambda^{=} x_{3}$ and $t \leq x_{1}$. Actually, $x_{2} \lambda t \lambda x_{3}$ since $\left\{x_{1}, x_{2}, x_{3}\right\}$ is an antichain. We obtain $x_{2} \lambda x_{1}$ from (A7), which contradicts $x_{1} \lambda x_{2}$ by (A5). This proves (3.3).

Case 1. Here we assume that there exists an element $u \in G \backslash F$ such that

$$
u \in \downarrow \operatorname{Min} \operatorname{Betw}(x, y) \backslash \operatorname{Min} \operatorname{Betw}(x, y)=\downarrow \operatorname{Min} \operatorname{Betw}(x, y) \backslash F
$$

and $u \not z z$ for some $z \in \operatorname{Min} \operatorname{Betw}(x, y)$. In what follows, $u$ will stand for such an element. The existence of $z$ implies $x \neq y$, and so $x \lambda y$. We claim that $u<x$ or $u<y$. Suppose the contrary. Then $x \not \leq u$ and $y \not \leq u$ by (3.4), so $x\|u, u\| y$, and there is a $t \in \operatorname{Min} \operatorname{Betw}(x, y)$ such that $u<t$. Since $x \lambda t \lambda y$, (A7) and its left-right dual combined with (A6) give $u \in \operatorname{Betw}(x, y) \subseteq F$, a contradiction. Hence, we can assume $u<x$. We claim $u \not \leq y$, and we prove this by way of contradiction. Suppose $u \leq y$. Since $u \| z$, either $u \lambda z \lambda y$ and (A2) yield $u \lambda y$, which contradicts $u \leq y$, or $x \lambda z \lambda u$ and we have $x \lambda u$, which contradicts $u<x$. Thus $u \not \leq y$. We know $u \nsupseteq y$ from $u \notin F$. If we had $y \lambda u$, then we would obtain $x \lambda u$ by (A2), which would contradict $u<x$. Therefore, $u \lambda y$, and $\langle u, y\rangle \in E(Q)$. Clearly, $F \subset \uparrow^{\text {hco }}\{u, y\} \subseteq G$, the minimality of $G$, and Lemma 3.1(i) give $G=\uparrow$ hco $\{u, y\}=\uparrow \operatorname{Min} \operatorname{Betw}(u, y)$.

We claim $\operatorname{Min} \operatorname{Betw}(u, y) \subseteq\{u\} \cup \uparrow \operatorname{Min} \operatorname{Betw}(x, y)$. Suppose the contrary. Then there exists a $t \in \operatorname{Min} \operatorname{Betw}(u, y)$ such that $u \neq t \notin \uparrow \operatorname{Min} \operatorname{Betw}(x, y)=F$. Observe $u \| t$. We have $x>t$, because $t \notin F$ excludes $x \lambda \leq t$ while $t \lambda x$ would lead to $t \lambda u$ by (A7), which would contradict $u \lambda t$ by (A5). Therefore, $G \supseteq \uparrow^{\text {hco }}\{t, y\} \supset$ $\uparrow^{\text {hco }}\{x, y\}=F$ and the minimality of $G$ imply that $G=\uparrow^{\text {hco }}\{t, y\}$. Using $u \in G$ and Lemma 3.1(i), we obtain an $s \in \operatorname{Betw}(t, y)$ such that $s \leq u$. Hence, (A7) yields $t \lambda u$ or $t \not \mid u$, which contradicts $u \lambda t$. Consequently, $\operatorname{Min} \operatorname{Betw}(u, y) \subseteq$ $\{u\} \cup \uparrow \operatorname{Min} \operatorname{Betw}(x, y)$.

Next, we claim that, for any $r \in Q^{+}$,

$$
r>u \Rightarrow r \in F .
$$

Suppose the contrary. That is, we have an $r \in G \backslash F$ such that $r>u$. The minimality of $G$ yields $u \in G=\uparrow^{\text {hco }}\{r, x, y\}$. Since $u \notin F=\uparrow^{\text {hco }}\{x, y\}$, (3.1) implies $u \in \uparrow^{\text {hco }}\{r, x\}$ or $u \in \uparrow^{\text {hco }}\{r, y\}$. If $r \| x$, then (3.2) excludes $u \in \uparrow^{\text {hco }}\{r, x\}$. If $r \not X x$, then $u \in \uparrow^{\text {hco }}\{r, x\}=\uparrow r \cup \uparrow x$ by Lemma 3.1(i), which is excluded by $u<r$ and $u<x$. Hence, $u \in \uparrow^{\text {hco }}\{r, y\}$. If $r \not \mid y$, then $u \in \uparrow^{\text {hco }}\{r, y\}=\uparrow r \cup \uparrow y$ by Lemma 3.1(i) again, which contradicts $u \| y$ and $u<r$. Thus $r \| y$, and $u \in \uparrow^{\text {hco }}\{r, y\}$ contradicts (3.2). This proves (3.5).

Finally, combining $\operatorname{Min} \operatorname{Betw}(u, y) \subseteq\{u\} \cup \uparrow \operatorname{Min} \operatorname{Betw}(x, y)=\{u\} \cup F,(3.5)$, and $G=\uparrow \operatorname{Min} \operatorname{Betw}(u, y)$, we obtain $G=F \cup\{u\}$, which gives $|G \backslash F|=1$.

Case 2. Here we assume that there exists an element $u \in G \backslash F$ such that $u \leq z$ for all $z \in \operatorname{Min} \operatorname{Betw}(x, y)$. (In particular, $u \in \downarrow \operatorname{Min} \operatorname{Betw}(x, y)$.) In what follows, $u$ will stand for such an element. The minimality of $G$ and Lemma 3.1(i) give $G=\uparrow^{\text {hco }} u=\uparrow u$. We claim

$$
u \prec z \text { for all } z \in \operatorname{Min} \operatorname{Betw}(x, y) \text {. }
$$


To show this by way of contradiction, suppose the contrary. Then there is a $v$ such that $u<v<z$. Since $z$ is a minimal element of $\operatorname{Betw}(x, y)$, Lemma 3.1(i) easily implies $v \notin F$. The minimality of $G$ gives $u \in G=\uparrow$ hco $\{x, y, v\}$. We apply (3.1) to $\uparrow^{\text {hco }}\{x, y, v\}$. Since $v \notin F=\uparrow^{\text {hco }}\{x, y\}=\uparrow^{\text {hco }} \operatorname{Min} \operatorname{Betw}(x, y)$, left-right symmetry allows us to assume $y \in \uparrow^{\text {hco }}\{x, v\}$. This gives $u \in G=\uparrow^{\text {hco }}\{x, v\}$. Now if we had $x \not \mid v$, then $u \in \uparrow^{\text {hco }}\{x, v\}=\uparrow^{\text {hco }}\{x\} \cup \uparrow^{\text {hco }}\{v\}=\uparrow x \cup \uparrow v$ would contradict $x>u$ and $v>u$. Otherwise $\langle x, v\rangle$ or $\langle v, x\rangle$ belongs to $E(Q)$, and $u \in \uparrow^{\text {hco }}\{x, v\}$ contradicts (3.2). This proves (3.6).

Next, we claim

$$
(\forall z \in \uparrow u)(z>u \Rightarrow z \in F) .
$$

Suppose the contrary, and pick a $v \in \uparrow u$ such that $v>u$ and $v \notin F=\uparrow^{\text {hco }}\{x, y\}$. The minimality of $G$ yields $u \in G=\uparrow^{\mathrm{hco}}\{x, y, v\}$. By (3.1), $v \notin F$, and left-right symmetry, we can assume $u \in \uparrow^{\text {hco }}\{x, v\}$. Since $u \prec x$ and $u<v$ exclude $x \not \mid v$, (3.2) yields the same contradiction as in the previous paragraph.

Finally, (3.7) and $G=\uparrow u$ imply $|G \backslash F|=1$.

Case 3. Here we assume that for all $u \in G \backslash F, u \notin \downarrow \operatorname{Min} \operatorname{Betw}(x, y)$. In what follows, $u$ will stand for such an element of $G \backslash F$. Since $u \notin F=\uparrow \operatorname{Min} \operatorname{Betw}(x, y)$, the primary assumption of the present case yields that $\{u\} \cup \operatorname{Min} \operatorname{Betw}(x, y)$ is an antichain and $u \notin \operatorname{Betw}(x, y)$. Hence either $u \lambda x$ or $y \lambda u$; we can assume the latter by left-right symmetry. Since $F=\uparrow^{\text {hco }}\{x, y\}$ is a proper subset of $\uparrow^{\text {hco }}\{x, u\}$ by (3.3) and $\uparrow^{\text {hco }}\{x, u\} \subseteq G$, the minimality of $G$ implies $G=\uparrow^{\text {hco }}\{x, u\}$. We claim that $u$ is immediately on the right of $y$, that is,

$$
\text { there is no } v \text { such that } y \lambda v \lambda u \text {. }
$$

To prove this by contradiction, suppose the contrary, and take such an element $v$. Since $x \lambda v \lambda u$ by (A2), we have $v \in G$. Also, $F=\uparrow^{\text {hco }}\{x, y\} \subseteq \uparrow^{\text {hco }}\{x, v\}$. But $v \notin F$ and $u \notin \uparrow$ hco $\{x, v\}$ by (3.3). Hence, $F \subset \uparrow^{\text {hco }}\{x, v\} \subset G$ contradicts the minimality of $G$. This proves (3.8). Next, we claim

$$
(\forall v \in Q)(u<v \Rightarrow v \in F) .
$$

Suppose the contrary. Then $F=\uparrow^{\text {hco }}\{x, y\} \subset \uparrow^{\text {hco }}\{x, y, v\} \subseteq G$, and the minimality of $G$ yields $G=\uparrow^{\text {hco }}\{x, y, v\}$. Since $v \notin F=\uparrow^{\text {hco }}\{x, y\}$, (3.1) implies $u \in G=\uparrow^{\text {hco }}\{x, v\}$ or $u \in \uparrow$ hco $\{y, v\}$. If $x \not y$, then $\uparrow^{\text {hco }}\{x, v\}=\uparrow x \cup \uparrow v$, and $u \| x$ and $u<v$ exclude $u \in \uparrow^{\text {hco }}\{x, v\}$. If $x \| v$, then $u \notin \uparrow^{\text {hco }}\{x, v\}$ by (3.2). Hence, $u \in \uparrow^{\text {hco }}\{y, v\}$. We can exclude $\left.y \not\right\}$ in the same way as we excluded $x \not v$ above. Hence $y \| v$, and (3.2) gives a contradiction. This proves (3.9).

Now we are in the position to show that $G=\uparrow^{\text {hco }}\{x, u\}$ equals $F \cup\{u\}$. The " $\supseteq$ " inclusion is clear. To prove the converse inclusion, assume $t \in G \backslash\{u\}$. By Lemma 3.1(i), there exists a $v \in \operatorname{Min} \operatorname{Betw}(x, u)$ such that $v \leq t$. If $v=u$, then $t \in F$ by (3.9). If $v \in F$, in particular, if $v=x$, then $t$ trivially belongs to $F$. Hence, for the sake of contradiction, suppose $v \notin F$ and $x \lambda v \lambda u$. We claim that there exists a $z \in \operatorname{Min} \operatorname{Betw}(x, y)$ such that $v<z$. Suppose the contrary, that is, $v \nless z$ for all $z \in \operatorname{Min} \operatorname{Betw}(x, y)$. Since $v \notin F$, we also have $v \nsucceq z$ for all $z \in \operatorname{Min} \operatorname{Betw}(x, y)$. Hence $\{v\} \cup \operatorname{Min} \operatorname{Betw}(x, y)$ is an antichain. Since $v \lambda u$, (3.8) excludes $y \lambda v$. However, $y \| v$, and we obtain $v \lambda y$. Hence, $v \in \operatorname{Betw}(x, y)$. This yields $v \in \uparrow^{\text {hco }}\{x, y\}=F$, which is a contradiction. Therefore, there exists a $z \in \operatorname{Min} \operatorname{Betw}(x, y)$ such that $v<z$. Thus, we have $v \in G \backslash F$ and 
$v \in \downarrow \operatorname{Min} \operatorname{Betw}(x, y)$. This is a contradiction, because we are dealing with Case 3 . This proves $G=F \cup\{u\}$ and $|G \backslash F|=1$.

An order filter $F$ of a quasiplanar diagram $Q$ is left-closed if for all $x \in F$ and $y \in Q, y \lambda x$ implies $y \in F$. Right-closed order filters $G$ are defined analogously by the property $(x \lambda y$ and $x \in G) \Rightarrow y \in G$. Clearly, left-closed and right-closed order filters are hco-filters. Definition 2.10(iv)-(v) should be kept in mind.

Lemma 3.6. If $Q$ is a quasiplanar diagram, then the definition of $\vec{F}=\vec{F}(Q)$ and that of $\vec{G}=\vec{G}(Q)$ make sense. The members of $\vec{F}$ are left-closed order filters, those of $\vec{G}$ are right-closed ones, and each element of the lattice $\left\langle F_{\mathrm{hco}}(Q) ; \leq^{d}\right\rangle$ is of the form $F_{i} \vee G_{j}$.

Proof. We prove by induction on $i$ that $F_{i}$ makes sense and it is a left-closed order filter of size $i+1$. This is obvious for $F_{0}=\{1\}$. Assume that $F_{n}$ is well-defined, it is a left-closed order filter, $\left|F_{n}\right|=n+1$, and $n+2 \leq|Q|-2$. Then $Q^{+} \backslash F_{n} \neq \varnothing$. Hence $\operatorname{Max}\left(Q \backslash F_{n}\right)$ is a antichain, which has a unique leftmost element $f_{n}$. We let $F_{n+1}=F \cup\left\{f_{n}\right\}$. It is an order filter, because $f_{n}$ is a maximal element outside $F_{n}$. Striving for a contradiction, suppose that $F_{n+1}$ is not left-closed. Then there is an $x \in Q^{+} \backslash F_{n}$ such that $x \lambda f_{n}$. By finiteness, there exists a $u \in \uparrow x \cap \operatorname{Max}\left(Q \backslash F_{n}\right)$. Since $x \| f_{n}$, we have $u \neq f_{n}$, which gives $f_{n} \lambda u$ by the definition of $f_{n}$. It follows from (A7) that $f_{n} \lambda x$, which contradicts $x \lambda f_{n}$. Consequently, $F_{n+1}$ is a left-closed order filter. This proves that $\vec{F}$ consists of well-defined left-closed order filters, and left-right duality yields that $\vec{G}$ consists of right-closed ones.

Next, let $B \in F_{\text {hco }}(Q)$. By Lemma $3.2, B=\uparrow^{\text {hco }}\{x, y\}$ for a unique $\langle x, y\rangle \in$ $E(Q)$. Let $i$ be the least subscript such that $y \in F_{i}$. Similarly, let $j$ be the smallest subscript such that $x \in G_{j}$. We claim $B=F_{i} \cap G_{j}$; in the lattice $\left\langle F_{\text {hco }}(Q) ; \leq^{d}\right\rangle$, this means $B=F_{i} \vee G_{j}$. Since $F_{i}$ is left-closed, $x \in F_{i}$. Similarly, $y \in G_{j}$ since $G_{j}$ is right-closed. Hence $\{x, y\} \subseteq F_{i} \cap G_{j}$, and we conclude $B=\uparrow$ hco $\{x, y\} \subseteq F_{i} \cap G_{j}$. In quest of a contradiction, suppose we have an element $z \in\left(F_{i} \cap G_{j}\right) \backslash B$. First, assume that $\{x, y, z\}$ is an antichain. (This antichain consists of two or three elements, depending on whether $x=y$ or $x \lambda y$.) Since $z \in \operatorname{Betw}(x, y)$ would imply $z \in B$, we have $z \lambda x$ or $y \lambda z$. If $y \lambda z$, then $z \in F_{i}$ implies $z \in F_{i} \backslash\{y\}=F_{i-1}$. However, then $y \in F_{i-1}$ since $F_{i-1}$ is left-closed, and this contradicts the definition of $i$. The case $z \lambda x$ contradicts the definition of $j$ similarly. Therefore, $\{x, y, z\}$ is not an antichain. Since $x \leq z$ and $y \leq z$ are excluded by $z \notin B$, we can assume $z<y$ by left-right symmetry. Then $z \in F_{i} \backslash\{y\}=F_{i-1}$. Since $F_{i-1}$ is an order-filter, we obtain $y \in F_{i-1}$, which contradicts the definition of $i$.

3.2. Statements on planar slim semimodular lattice diagrams. Let $D$ be a planar lattice diagram. If $a \leq b \in D$, then the interval $[a, b]$ determines a subdiagram, which is denoted by $[a, b]_{D}$ or, if there is no danger of confusion, by $[a, b]$. An element of $D$ is a narrows of $D$ if it is comparable with every element of $D$. The set of narrows is denoted by $\operatorname{Nar}(D)$. The vertical mirror image $\operatorname{VFlip}(D)$ was defined right after Corollary 2.12. We need the following statement, which is somewhat stronger than Lemma 2.7.

Lemma 3.7 (Czédli and Schmidt [20, Lemma 4.7] or, more explicitly, Czédli and Grätzer [15]). Let $D$ and $E$ be finite planar slim semimodular lattice diagrams, and let $\operatorname{Nar}(D)=\left\{0=d_{0}<d_{1}<\cdots<d_{m}=1\right\}$ and $\operatorname{Nar}(E)=\left\{0=e_{0}<e_{1}<\cdots<\right.$ 


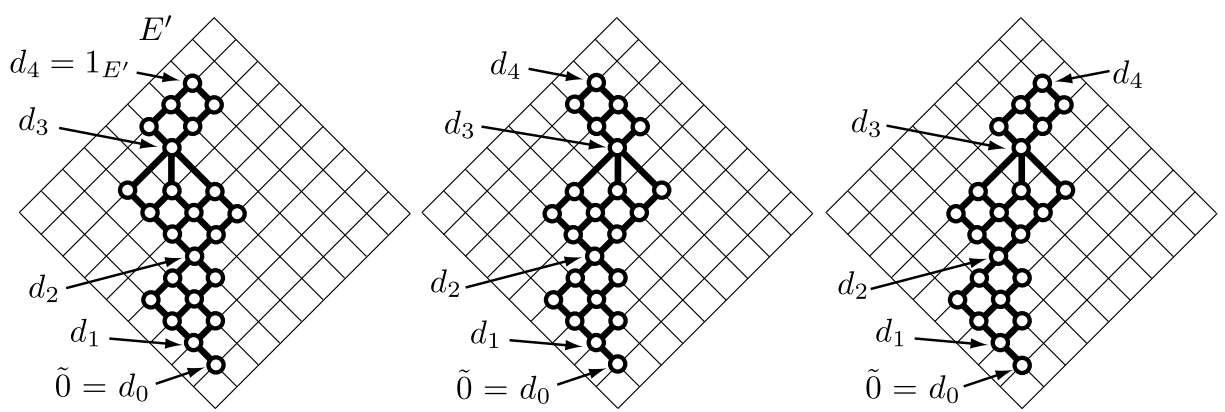

Figure 5 . Replacing $\left[d_{2}, d_{3}\right]$ by $\operatorname{VFlip}\left(\left[d_{2}, d_{3}\right]\right)$

$\left.e_{n}=1\right\}$. Then $D$ and $E$ determine isomorphic lattices if and only if $m=n$ and, up to similarity, $\left[d_{i-1}, d_{i}\right]_{D} \in\left\{\left[e_{i-1}, e_{i}\right]_{E}, \operatorname{VFlip}\left(\left[e_{i-1}, e_{i}\right]_{E}\right)\right\}$ for $i=1, \ldots, n$.

Next, we recall some well-known facts; see, for example, Kelly and Rival [27, Proposition 5.2] and Czédli and Grätzer [15, Exercises 3.5 and 3.7]. The order dimension of a poset $P=\langle P ; \leq\rangle$ is the least $n$ such that the ordering relation " $\leq$ " is the intersection of $n$ linear (that is, chain) orderings. Equivalently, it is the least $n$ such that $P$ can be order-embedded into the direct product of $n$ chains. By a grid we mean a planar diagram of a direct product of two nontrivial finite chains such that every edge is of slope $45^{\circ}$ or $135^{\circ}$. (A chain is nontrivial if it has at least two elements.) A finite lattice has a planar diagram iff it is of order-dimension at most 2. Therefore, each planar lattice has a planar diagram $D$ that is embedded in a grid $G$ such that the vertices of $D$ are also vertices of $G$ and, in addition, for $a, b \in D$, we have $a \leq b$ in $D$ iff $a \leq b$ holds in $G$. Figures 5 and 6 give examples for such embeddings. If $D$ is embedded in a grid and $a, b \in D$ are distinct elements, then $a<b$ if and only if the vector from $a$ to $b$ is of slope between $45^{\circ}$ and $135^{\circ}$. When we deal with diagrams from the aspect of embeddability in grids, we cannot consider similar diagrams equal. For example, while $D_{9}$ in Figure 7 and also the lattice diagrams $D_{1}, \ldots, D_{8}$ in Figures 1,3 , and 4 can be embedded in grids, $D_{10}$ in Figure 7 is similar to $D_{9}$ but $D_{10}$ cannot be embedded in a grid. We need the following statement.

Lemma 3.8. If $D$ is a lattice diagram embedded in a grid, then $D$ is a planar diagram.

Proof. The idea is taken from Kelly and Rival [27, Proposition 5.2]; see also Czédli and Grätzer [15, Exercise 3.7]. Suppose, for a contradiction, that $a_{1} \prec b_{1}$ and $a_{2} \prec b_{2}$ are edges of $D$ with a forbidden "non-planar" intersection. Clearly, $a_{1} \neq a_{2}$ and $b_{1} \neq b_{2}$. Since the slope of the line through $a_{i}$ and $b_{j}$ is between $45^{\circ}$ or $135^{\circ}$, we obtain $a_{i} \leq b_{j}$ for all $i, j \in\{1,2\}$. Hence, $a_{1}<a_{1} \vee a_{2} \leq b_{1} \wedge b_{2}<b_{1}$, which contradicts $a_{1} \prec b_{1}$.

Now we are ready to state and prove the following lemma.

Lemma 3.9. If $D$ is a finite planar slim semimodular lattice diagram, then $\alpha(D)$ defined in Definition 2.8 exists (and it is a quasiplanar diagram).

Proof. Let $D^{\prime}$ and $L^{\prime}$ denote the diagram and the lattice we obtain from $D$ and $L$, respectively, by adding a new bottom element $\tilde{0}$. Since $D$ has a least element, $D^{\prime}$ is 
a planar diagram, and it is a diagram of $L^{\prime}$. Hence, $L^{\prime}$ is a planar lattice, so it has a diagram $E^{\prime}$ embedded into a grid $G$; see Figure 5 on the left. By Lemma 3.8, $E^{\prime}$ is a planar diagram. The points of $G$ are the intersections of the thin lines, and $E^{\prime}$ consists of the little circles and the thick lines. Let $\operatorname{Nar}\left(L^{\prime}\right)=\left\{\tilde{0}=d_{0}<d_{1}<\cdots<\right.$ $\left.d_{m}=1\right\}=\operatorname{Nar}\left(E^{\prime}\right)$. We can assume that $G$ is large enough in the sense that the elements of $E^{\prime}$ are sufficiently far from the boundary of $G$. In order to see that we can replace the subdiagram $\left[d_{i-1}, d_{i}\right]$ by its vertical mirror image VFlip $\left(\left[d_{i-1}, d_{i}\right]\right)$ so that the new diagram is still a part of the same grid, see Figure 5 with $i=3$ and $m=4$, we do the following. First, by reflecting a square-shaped subgrid with bottom $d_{i-1}$ that contains $1_{E^{\prime}}$, we reflect $\left[d_{i-1}, d_{m}\right]=\left[d_{i-1}, 1_{E^{\prime}}\right]$. Second, we reflect $\left[d_{i}, d_{m}\right]$ together with a square-shaped subgrid with bottom $d_{i}$ similarly. Since $G$ is large enough, we can vertically reflect $\left[d_{i-1}, d_{i}\right]$ for several values of $i$, one by one. We know that $D^{\prime}$ and $E^{\prime}$ determine the same lattice, $L^{\prime}$. Therefore, Lemma 3.7 allows us to assume that $E^{\prime}=D^{\prime}$.

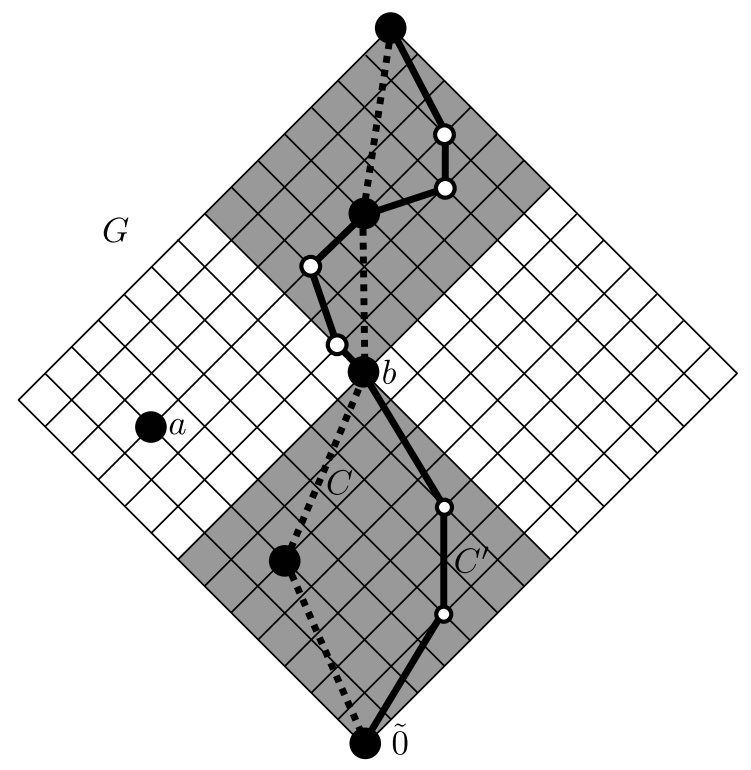

FIgURE 6. Illustrating the proof of Lemma 3.9

According to (i) without its parenthesized part and (ii) of Definition 2.8, we obtain a diagram $Q$ of $\left\{1_{D^{\prime}}\right\} \cup \operatorname{Mi} D^{\prime}=\left\{\tilde{0}, 1_{L}\right\} \cup \operatorname{Mi} L=\left\{\tilde{0}, 1_{D}\right\} \cup \operatorname{Mi} D$, which is a subposet of $D^{\prime}$, in the following straightforward way: keep the position of its elements in the plane, delete the elements of $D^{\prime} \backslash\left(\left\{\tilde{0}, 1_{D}\right\} \cup \mathrm{Mi} D\right)$ and all edges of $D^{\prime}$, and add a straight line segment from $a$ to $b$ whenever $b$ covers $a$ in the subposet. In Figure $6, D^{\prime}$ consists of the (empty and black-filled) circles and the thick solid lines. The elements and the edges of $Q$ are denoted by black-filled circles and thick dotted lines, respectively. Only a part of $D^{\prime}$ and a part of $Q$ are depicted.

We have to show that $Q$ is quasiplanar and that it satisfies (iii) of Definition 2.8. The fact that the elements of $Q \backslash\left\{0_{Q}, 1_{Q}\right\}$ are meet-irreducible in $D^{\prime}$ will not be used. Hence, for later reference, we note that

no matter which elements of $G$ constitute $Q$, the proof below (with $D^{\prime}=G$ ) will yield that $Q$ is quasiplanar. 
Let $a, b \in Q$ such that $a \| b$ in $G$. Then $a \| b$ in $Q$, and we claim that

if $a \lambda_{G} b$, then $a \lambda_{D^{\prime}} b$ and $a$ is on the left of every chain through $b$ in $Q$.

Assume $a \lambda_{G} b$, and consider a maximal chain $C^{\prime}$ through $b$ in $D^{\prime}$; it consists of the thick solid lines. Let $S=\{x \in G: x \nmid b\}$; it is the grey area in the figure. We know that $a \notin S$. Furthermore, $a$ is on the left of the grey area $S$, because it is on the left of the left boundary chain of $S$ by the definition of $\lambda_{G}$. Since $C^{\prime} \subseteq S$ and $D^{\prime}$ is a planar lattice diagram, Lemma 2.3 and the definition of $\lambda_{D^{\prime}}$ yield $a \lambda_{D^{\prime}} b$. Next, let $C$ be an arbitrary maximal chain through $b$ in $Q$; it consists of the thick dotted lines. Since $C \subseteq S, a$ is on the left of $C$ in $Q$. This proves (3.11), and we similarly obtain that

if $a \varrho_{G} b$ and $a, b \in Q$, then $a \varrho_{D^{\prime}} b$ and $a$ is on the right of every chain through $b$ in $Q$.

Since $G$ is a planar lattice diagram, it is quasiplanar by Lemma 2.6. Applying (A5) and (A6) to $G$ and to $a, b \in Q$ with $a \| b$, we obtain that exactly one of the possibilities $a \lambda_{G} b$ and $a \varrho_{G} b$ holds. Hence, (3.11) and (3.12) imply that (A1) holds in $Q$. From (3.11) and (3.12) we also obtain that, for $a, b \in Q$,

$$
a \lambda_{D^{\prime}} b \Longleftrightarrow a \lambda_{Q} b \text { and } a \varrho_{D^{\prime}} b \Longleftrightarrow a \varrho_{Q} b .
$$

Thus, (A2) and (A3) hold in $Q$ since they are valid in $D^{\prime}$ by Lemma 2.6. Therefore, $Q$ is a quasiplanar diagram. In (3.13), we can replace $\lambda_{D^{\prime}}$ and $\varrho_{D^{\prime}}$ by $\lambda_{D}$ and $\varrho_{D}$, respectively, since $a \| b$ excludes $\tilde{0} \in\{a, b\}$. With this modification, (3.13) yields $\alpha(D)=Q$.

As a consequence of the proof above, we conclude the following statement. It explains how the $\alpha\left(D_{i}\right)=Q_{i}$, for $i \in\{1 \ldots, 9\}$, were drawn in our figures.

Corollary 3.10. If a finite planar slim semimodular lattice diagram $D$ is embedded in a grid, then we can obtain $\alpha(D)$ in the following three steps:

(i) If $0_{D} \in \mathrm{Mi} D$, then add a new zero to obtain $D^{\prime}$, which is also embedded in the same grid. Otherwise, let $D^{\prime}=D$.

(ii) Keep the vertices belonging to $\left\{0_{D^{\prime}}, 1_{D^{\prime}}\right\} \cup \mathrm{Mi} D^{\prime}$, which are concrete points in the plane, and delete the rest of vertices.

(iii) Draw the edges according to the restriction of the ordering of $D^{\prime}$.

The case $0_{D} \in \mathrm{Mi} D$ is illustrated by $D_{7}$ in Figure 3. Now we import two statements from Czédli [11]. We say that $y$ is horizontally between $x_{0}$ and $x_{1}$ if $x_{0} \lambda y \lambda x_{1}$ or $x_{1} \lambda y \lambda x_{0}$. Note that $\left\{x_{0}, x_{1}, y\right\}$ is a 3 -element antichain in this case.

Lemma 3.11 (Czédli [11, Proposition 3.13]). Let $D$ be a finite planar lattice diagram, and let $\left\{x_{0}, x_{1}, y\right\}$ be a 3-element antichain in $D$. Then the following two statements hold.

(i) If $y$ is horizontally between $x_{0}$ and $x_{1}$, then $x_{0} \wedge x_{1} \leq y$.

(ii) If, in addition, $D$ is slim and semimodular and $x_{0} \wedge x_{1} \leq y$, then $y$ is horizontally between $x_{0}$ and $x_{1}$.

Lemma 3.12 (Czédli [11, Proposition 3.14]). If $L$ is a finite semimodular lattice, $a \in \operatorname{Mi} L, b, c \in L, a<c$, and $b \wedge c \leq a$, then $b \leq a$. 
The following lemma is a particular case of Czédli and Schmidt [17, Lemma 2.2]. The left boundary chain and the right boundary chain of a planar lattice diagram $D$ are denoted by $\mathrm{C}_{\mathrm{l}}(D)$ and $\mathrm{C}_{\mathrm{r}}(D)$, respectively.

Lemma 3.13 ([17]). Let $C_{1}$ and $C_{2}$ be maximal chains in a finite slim semimodular lattice $L$ such that Ji $L \subseteq C_{1} \cup C_{2}$. Then $L$ has a planar diagram $D$ such that $C_{1}=$ $\mathrm{C}_{\mathrm{l}}(D)$ and $C_{2}=\mathrm{C}_{\mathrm{r}}(D)$. Furthermore, this diagram is unique (up to similarity).

\subsection{Join and meet representations in slim semimodular lattices.}

Definition 3.14. For $x$ in a planar lattice diagram $D$, the largest element of $\downarrow x \cap \mathrm{C}_{\mathrm{l}}(D)$ and that of $\downarrow x \cap \mathrm{C}_{\mathrm{r}}(D)$ are the left support of $x$, denoted by $\operatorname{lsp}(x)$, and the right support of $x$, denoted by $\operatorname{rsp}(x)$, respectively.

It follows from the definition of slimness that

$$
x=\operatorname{lsp}(x) \vee \operatorname{rsp}(x) \text {, for all } x \in D,
$$

provided $D$ is a planar slim lattice diagram.

Lemma 3.15. For $x \| y$ in a planar slim semimodular lattice diagram $D$, we have $x \lambda y$ iff $\operatorname{lsp}(x)>\operatorname{lsp}(y)$ and $\operatorname{rsp}(x)<\operatorname{rsp}(y)$. Furthermore, $x \leq y$ iff $\operatorname{lsp}(x) \leq \operatorname{lsp}(y)$ and $\operatorname{rsp}(x) \leq \operatorname{rsp}(y)$

Proof. If $\operatorname{lsp}(x)=\operatorname{lsp}(y)$, then $\operatorname{rsp}(x) \not X \operatorname{rsp}(y)$ since $\mathrm{C}_{\mathrm{r}}(D)$ is a chain and (3.14) gives $x \not K y$. Hence, $x \| y$ implies $\operatorname{lsp}(x) \neq \operatorname{lsp}(y)$ and $\operatorname{rsp}(x) \neq \operatorname{rsp}(y)$.

Assume $x \lambda y$. Striving for a contradiction, $\operatorname{suppose} \operatorname{lsp}(x) \ngtr \operatorname{lsp}(y)$. We know that $\operatorname{lsp}(x)$ and $\operatorname{lsp}(y)$ are comparable, since $\mathrm{C}_{1}(D)$ is a chain, and they are distinct, since $x \| y$. Hence, $\operatorname{lsp}(x)<\operatorname{lsp}(y)$. By the definition of $\operatorname{lsp}(x)$, we have $\operatorname{lsp}(y) \not \leq x$. On the other hand, $x \| y \geq \operatorname{lsp}(y)$ implies $\operatorname{lsp}(y) \geq x$. That is, $\operatorname{lsp}(y) \| x$. Since $x$, like any element, is on the right of $\mathrm{C}_{1}(D)$ and $\operatorname{lsp}(y) \in \mathrm{C}_{1}(D)$, the left-right dual of Lemma 2.3 yields $x \varrho \operatorname{lsp}(y)$, that is, $\operatorname{lsp}(y) \lambda x$. Since $D$ is quasiplanar by Lemma 2.6 and $x \lambda y$, (A2) yields $\operatorname{lsp}(y) \lambda y$. This contradicts $\operatorname{lsp}(y) \leq y$. Therefore, $x \lambda y$ implies $\operatorname{lsp}(x)>\operatorname{lsp}(y)$. By left-right duality, it also implies $\operatorname{rsp}(x)<\operatorname{rsp}(y)$. This proves the "only if" part of the lemma.

To prove the "if" part, assume $\operatorname{lsp}(x)>\operatorname{lsp}(y)$ and $\operatorname{rsp}(x)<\operatorname{rsp}(y)$. Clearly, $x \| y$. We cannot have $y \lambda x$ since it would contradict the "only if" part. Thus, $x \lambda y$. Finally, the second statement of the lemma is obvious.

As a counterpart of Definition 3.14, we present the following concept.

Definition 3.16. Let $D$ be a finite planar slim semimodular lattice diagram, and let $b \in D \backslash\{1\}$. The left upper support and the right upper support of $b$, denoted by $b^{\text {lus }}$ and $b^{\text {rus }}$, are the leftmost and the rightmost element of the antichain $\operatorname{Min}(\uparrow b \cap \operatorname{Mi} D)$, respectively.

A meet $x_{1} \wedge \cdots \wedge x_{n}$ in a lattice is irredundant if

$$
x_{1} \wedge \cdots \wedge x_{i-1} \wedge x_{i+1} \wedge \cdots \wedge x_{n} \neq x_{1} \wedge \cdots \wedge x_{n}
$$

for $i=1, \ldots, n$.

Lemma 3.17. Let $D$ be a finite planar slim semimodular lattice diagram, and let $b \in D \backslash\{1\}$. Then $b=b^{\text {lus }} \wedge b^{\text {rus }}$. Furthermore, if $X \subseteq$ Mi $L$ such that $b=\bigwedge X$ is an irredundant meet representation of $b$, then $X=\left\{b^{\text {lus }}, b^{\text {rus }}\right\}$. 
Proof. Obviously, $b=\bigwedge \operatorname{Min}(\uparrow b \cap \operatorname{Mi} D)$. Lemma 3.11(i) implies $b=b^{\text {lus }} \wedge b^{\text {rus }}$. If $b^{\text {lus }} \neq b^{\text {rus }}$, then $b^{\text {lus }} \| b^{\text {rus }}$ and $b=b^{\text {lus }} \wedge b^{\text {rus }}$ is an irredundant meet representation. Hence, with the notation $Y=\left\{b^{\text {lus }}, b^{\text {rus }}\right\}, b=\wedge Y$ is an irredundant meet-representation, even if $b^{\text {lus }}=b^{\text {rus }}$. Since slim semimodular lattices are joindistributive, see Czédli, Ozsvárt, and Udvari [16, Corollary 2.2], and the irredundant meet-representations in a join-distributive lattice are unique by Dilworth [21], the rest of the lemma follows.

As a counterpart of Lemma 3.15, we have the following.

Lemma 3.18. Let $x$ and $y$ be elements of a planar slim semimodular lattice diagram $D$. Then the following two assertions hold.

(i) $x \leq y$ iff $x^{\text {lus }} \lambda^{\leq} y^{\text {lus }}$ and $x^{\text {rus }} \varrho^{\leq} y^{\text {rus }}$;

(ii) $x \lambda y$ iff $x^{\text {lus }} \lambda^{<} y^{\text {lus }}$ and $x^{\text {rus }} \lambda^{>} y^{\text {rus }}$.

Proof. We shall often use the identity $b=b^{\text {lus }} \wedge b^{\text {rus }}$ of Lemma 3.17 without further reference.

To prove the "only if" part of (i), assume $x \leq y$. By left-right symmetry, it suffices to prove $x^{\text {lus }} \lambda \leq y^{\text {lus }}$. If $x^{\text {lus }}=x^{\text {rus }}$, then $x^{\text {lus }}=x \leq y \leq y^{\text {lus }}$ gives that $x^{\text {lus }} \lambda \leq y^{\text {lus }}$. Hence, we also assume $x^{\text {lus }} \lambda x^{\text {rus }}$. We claim that

$$
y^{\text {lus }} \nless x^{\text {lus }}, \quad y^{\text {lus }} \nless x^{\text {rus }}, \quad y^{\text {rus }} \nless x^{\text {lus }}, \quad y^{\text {rus }} \nless x^{\text {rus }} .
$$

Suppose, for a contradiction, that $y^{\text {lus }}<x^{\text {lus }}$. Then $y^{\text {lus }} \neq x$ since $x$ is meetreducible. Hence, $x<y^{\text {lus }}$, and $x=y^{\text {lus }} \wedge x^{\text {rus }}$ is an irredundant meet representation of $x$, different from $x=x^{\text {lus }} \wedge x^{\text {rus }}$. This is impossible by Lemma 3.17. This proves $y^{\text {lus }} \nless x^{\text {lus }}$, and the rest of (3.15) follows similarly.

If $y^{\text {lus }}=y^{\text {rus }}$ and $\left\{x^{\text {lus }}, y, x^{\text {rus }}\right\}$ is a 3 -element antichain, then we obtain $x^{\text {lus }} \lambda \leq$ $y=y^{\text {lus }}$ from Lemma 3.11(ii). So, if $y^{\text {lus }}=y^{\text {rus }}$, then we can assume that $\left\{x^{\text {lus }}, y, x^{\text {rus }}\right\}$ is not a 3 -element antichain. By (3.15), if $x^{\text {lus }} \nVdash y=y^{\text {lus }}$, then $x^{\text {lus }} \lambda \leq y^{\text {lus }}$ trivially holds. Hence, taking (3.15) into account, we may assume that $x^{\text {lus }} \| y$ and $y=y^{\text {rus }} \geq x^{\text {rus }}$. Since $x^{\text {lus }} \lambda x^{\text {rus }}$ and since (3.15) excludes $y=y^{\text {lus }}<x^{\text {lus }}$, (A7) yields $x^{\text {lus }} \lambda \leq y^{\text {lus }}$.

Therefore, we may assume that $y^{\text {lus }} \neq y^{\text {rus }}$, so $y^{\text {lus }} \lambda y^{\text {rus }}$. We know that $x^{\text {lus }} \wedge x^{\text {rus }} \leq y \leq y^{\text {lus }}$. If $\left\{x^{\text {lus }}, y^{\text {lus }}, x^{\text {rus }}\right\}$ is a 3 -element antichain, then $x^{\text {lus }} \lambda \leq y^{\text {lus }}$ follows from Lemma 3.11(ii). If $x^{\text {lus }} \nVdash y^{\text {lus }}$, then we obtain $x^{\text {lus }} \lambda^{\leq} y^{\text {lus }}$ from (3.15). Finally, if $x^{\text {rus }} \nVdash y^{\text {lus }}$, then (A7) and (3.15) imply $x^{\text {lus }} \lambda^{\leq} y^{\text {lus }}$. We have proved the "only if" part of (i).

To prove the "if" part, assume $x^{\text {lus }} \lambda^{\leq} y^{\text {lus }}$ and $x^{\text {rus }} \varrho^{\leq} y^{\text {rus }}$. If $x^{\text {lus }} \lambda y^{\text {lus }}$ and $x^{\text {rus }} \varrho y^{\text {rus }}$, then $x^{\text {lus }} \lambda y^{\text {lus }} \lambda^{=} y^{\text {rus }} \lambda x^{\text {rus }}$ and Lemma 3.11(i) imply $x=$ $x^{\text {lus }} \wedge x^{\text {rus }} \leq y^{\text {lus }}$ and $x \leq y^{\text {rus }}$, and we obtain $x \leq y$. If $x^{\text {lus }} \leq y^{\text {lus }}$ and $x^{\text {rus }} \leq y^{\text {rus }}$, then $x \leq y$ trivially follows. There are two more cases; we only deal with one of them, because the other one will follow by left-right duality. Assume $x^{\text {lus }} \leq y^{\text {lus }}$ and $x^{\text {rus }} \varrho y^{\text {rus }}$. If $x^{\text {lus }} \not 1 y^{\text {rus }}$, then $y^{\text {lus }} \ngtr y^{\text {rus }}$ excludes $x^{\text {lus }}>y^{\text {rus }}$, so we have $x^{\text {lus }} \leq y^{\text {rus }}$, and thus $x \leq x^{\text {lus }} \leq y^{\text {lus }} \wedge y^{\text {rus }}=y$. If $x^{\text {lus }} \| y^{\text {rus }}$, then $y^{\text {rus }}$ cannot be on the left of a maximal chain through $\left\{x^{\text {lus }}, y^{\text {lus }}\right\}$, because otherwise $y^{\text {rus }} \lambda y^{\text {lus }}$ by (A1), and so $y^{\text {rus }}=y^{\text {lus }} \geq x^{\text {lus }}$ is a contradiction. Hence, $y^{\text {rus }}$ is on the right of this chain, and we have $y^{\text {rus }} \varrho x^{\text {lus }}$, that is, $x^{\text {lus }} \lambda y^{\text {rus }}$. Since we also have $y^{\text {rus }} \lambda x^{\text {rus }}$, we obtain $x=x^{\text {lus }} \wedge x^{\text {rus }} \leq y^{\text {rus }}$ from Lemma 3.11(ii). Therefore, we conclude $x=x^{\text {lus }} \wedge x \leq y^{\text {lus }} \wedge y^{\text {rus }}=y$ again. This proves (i). 
Next, to prove the "only if" part of (ii), assume $x \lambda y$. By left-right duality, it suffices to prove $x^{\text {lus }} \lambda^{<} y^{\text {lus }}$. Assume first that $x^{\text {lus }}=x^{\text {rus }}=x$. Suppose, for a contradiction, that $x=x^{\text {lus }} \lambda^{<} y^{\text {lus }}$ fails. We have $x \nsupseteq y^{\text {lus }}$ and $x \nsupseteq y^{\text {rus }}$, because $x \nsupseteq y$. Hence, the failure of $x \lambda^{<} y^{\text {lus }}$ means that $y^{\text {lus }} \lambda x$. If $x \nVdash y^{\text {rus }}$ or $y^{\text {rus }} \lambda x$, then we can apply Lemma 2.2 to a maximal chain $C_{0}$ through $\left\{x, y^{\text {rus }}\right\}$ or through $x$ to conclude that $y=y^{\text {lus }} \wedge y^{\text {rus }}$ is on the left of $C_{0}$. However, $y$ is also on the right of this chain, because of $y \varrho x \in C_{0}$. Hence, $y \in C_{0}$, which contradicts $x \| y$. Thus, neither $x \nVdash y^{\text {rus }}$, nor $y^{\text {rus }} \lambda x$. Hence, $y^{\text {rus }} \varrho x$, and we have $y^{\text {lus }} \lambda x \lambda y^{\text {rus }}$. Applying Lemma 3.11(i), we obtain $y=y^{\text {lus }} \wedge y^{\text {rus }} \leq x$, which contradicts $x \lambda y$. Consequently, if $x^{\text {lus }}=x^{\text {rus }}$, then $x^{\text {lus }} \lambda^{<} y^{\text {lus }}$ holds. Therefore, we may assume $x^{\text {lus }} \lambda x^{\text {rus }}$.

Striving for a contradiction, suppose $x^{\text {lus }} \geq y^{\text {lus }}$. Extend the chain $\left\{y \leq y^{\text {lus }} \leq\right.$ $\left.x^{\text {lus }}\right\}$ to a maximal chain $C_{1}$. Since $x \lambda y, x$ is on the left of $C_{1}$. On the other hand, $x^{\text {rus }} \varrho x^{\text {lus }} \in C_{1}$ and Lemma 2.2 give that $x=x^{\text {lus }} \wedge x^{\text {rus }}$ is on the right of $C_{1}$. Hence, $x \in C_{1}$, which contradicts $x \| y$. Therefore, $x^{\text {lus }} \nsucceq y^{\text {lus }}$.

Next, for the sake of a contradiction, suppose $y^{\text {lus }} \lambda x^{\text {lus }}$. Extend $\left\{x, x^{\text {lus }}\right\}$ to a maximal chain $C_{2}$. Since $y^{\text {lus }} \lambda x^{\text {lus }}, y^{\text {lus }}$ is on the left of $C_{2}$, while $x \lambda y$ yields that $y$ is on the right of $C_{2}$. Hence Lemma 2.1 applies, and we obtain an element $z \in C_{2}$ such that $y \leq z \leq y^{\text {lus }}$. Since $z \not x$, as both belong to $C_{2}$, and $x \ngtr y$, we have $x<z$, and thus $x<y^{\text {lus }}$. Therefore, the set $\uparrow x \cap \downarrow y^{\text {lus }} \cap \operatorname{Mi} D$ is nonempty, since it contains $y^{\text {lus }}$. Let $t$ be a minimal element of this set. Clearly, $t$ belongs to the antichain $\operatorname{Min}(\uparrow x \cap \operatorname{Mi} D)$. Since $x^{\text {lus }}$ is the leftmost element of this antichain, we have $x^{\text {lus }} \lambda^{=} t$. We cannot have $x^{\text {lus }}=t$, because otherwise $x^{\text {lus }}=t \leq y^{\text {lus }}$ would contradict $y^{\text {lus }} \lambda x^{\text {lus }}$. Hence $x^{\text {lus }} \lambda t$. Now extend $\left\{t, y^{\text {lus }}\right\}$ to a maximal chain $C_{3}$. Then $x^{\text {lus }}$ is on the left of $C_{3}$ since $x^{\text {lus }} \lambda t$, and $x^{\text {lus }}$ is also on the right of $C_{3}$ since $y^{\text {lus }} \lambda x^{\text {lus }}$. Therefore, $x^{\text {lus }} \in C_{3}$ and so $x^{\text {lus }} \nVdash y^{\text {lus }}$, which contradicts $y^{\text {lus }} \lambda x^{\text {lus }}$. This proves that $y^{\text {lus }} \lambda x^{\text {lus }}$ is impossible.

Now that we have excluded the other two possibilities, we conclude that $x \lambda y$ implies $x^{\text {lus }} \lambda^{<} y^{\text {lus }}$. This proves the "only if" part of (ii).

Finally, to prove the " "if" part of (ii), assume $x^{\text {lus }} \lambda^{<} y^{\text {lus }}$ and $x^{\text {rus }} \lambda^{>} y^{\text {rus }}$. Part (i) excludes $x \not y y$, and the "only if" part of (ii) excludes $y \lambda x$. Hence, $x \lambda y$.

\subsection{Further auxiliary statements.}

Lemma 3.19. If $Q$ is a quasiplanar diagram, then

$$
\operatorname{Mi}\left\langle F_{\text {hco }}(Q) ; \leq^{d}\right\rangle=\{\uparrow x: x \in Q \backslash\{0,1\}\} .
$$

Proof. Let $F \in F_{\text {hco }}(Q)$. By Lemma 3.2, $F$ is of the form $F=\uparrow^{\text {hco }}\{x, y\}$, where $x=\operatorname{lbe}(F), y=\operatorname{rbe}(F)$, and $x \lambda^{=} y \in Q^{+}$. First, assume $x=y$. Then $F=\uparrow x$ by Lemma 3.1(i). Clearly, $F \backslash\{x\} \in F_{\text {hco }}(Q)$, and it is the unique lower cover of $F$ with respect to set inclusion. Hence, $F \backslash\{x\}$ is the unique upper cover of $F$ in the lattice $\left\langle F_{\text {hco }}(Q) ; \leq^{d}\right\rangle$. That is, $F \in \operatorname{Mi}\left\langle F_{\text {hco }}(Q) ; \leq^{d}\right\rangle$, proving the " $\supseteq$ " part of the lemma. Next, assume $x \neq y$. Obviously, $F \neq \uparrow x$ and $F \neq \uparrow y$. By Lemma 3.1(i), $\uparrow x, \uparrow y \in F_{\text {hco }}(Q)$. Clearly, $F=\uparrow x \vee \uparrow y$ in the dual lattice $\left\langle F_{\text {hco }}(Q) ; \subseteq\right\rangle$. Thus $F=\uparrow x \wedge \uparrow y$ in $\left\langle F_{\text {hco }}(Q) ; \leq^{d}\right\rangle$, and $F \notin \operatorname{Mi}\left\langle F_{\text {hco }}(Q) ; \leq^{d}\right\rangle$. This proves $\not \supset$.

Lemma 3.20. For a quasiplanar diagram $Q, \beta_{2}(Q)$ makes sense, it is uniquely defined, and it is a planar diagram of a slim semimodular lattice. 
Proof. We know from Lemma 3.5 that $\left\langle F_{\text {hco }}(Q) ; \leq^{d}\right\rangle$ is a semimodular lattice. We obtain from Lemma 3.6 that the join-irreducible elements of this lattice belong to $\vec{F}(Q) \cup \vec{G}(Q)$. By Lemma 3.13, there exists a planar diagram $D$ of the lattice $\left\langle F_{\text {hco }}(Q) ; \leq^{d}\right\rangle$ such that $\mathrm{C}_{\mathrm{l}}(D)=\vec{F}(Q)$ and $\mathrm{C}_{\mathrm{r}}(D)=\vec{G}(Q)$. According to Definition $2.10(\mathrm{vi})$, this $D$ is $\beta_{2}(Q)$. The uniqueness of $\beta_{2}(Q)$ follows from Lemma 3.13.

Lemma 3.21. Let $Q$ be a quasiplanar diagram, and let $x, y \in Q$. Then $x \lambda y$ in $Q$ iff $\uparrow x \lambda \uparrow y$ in $\beta_{2}(Q)$.

Proof. To prove the "only if" part, assume $x \lambda y$, and let $n$ be the smallest subscript such that $y \in F_{n}$. Note that $y \in F_{n}$ iff $\uparrow y \subseteq F_{n}$ iff $F_{n} \leq d \uparrow y$. Note also that $n>k$ iff $F_{n} \leq^{d} F_{k}$. Therefore, since $\left.\mathrm{C}_{\mathrm{l}}\left(\beta_{2}(Q)\right)\right)=\vec{F}(Q)$ and $\left.\mathrm{C}_{\mathrm{r}}\left(\beta_{2}(Q)\right)\right)=\vec{G}(Q)$ by Definition 2.10(vi), $F_{n}=\operatorname{lsp}(\uparrow y)$; see Definition 3.14. Also, if $m$ is the smallest subscript such that $x \in F_{m}$, then $F_{m}=\operatorname{lsp}(\uparrow x)$. Since $F_{n}$ is left-closed, $x \in F_{n}$, which implies $m \leq n$. In fact, $m<n$ since $x \neq y$ yields $m \neq n$. Thus $\operatorname{lsp}(\uparrow x)=$ $F_{m}>^{d} F_{n}=\operatorname{lsp}(\uparrow y)$. Left-right duality yields $\operatorname{rsp}(\uparrow x)<^{d} \operatorname{rsp}(\uparrow y)$. Therefore, Lemma 3.15 yields $\uparrow x \lambda \uparrow y$ in $\beta_{2}(Q)$. This proves the "only if" part.

Conversely, assume $\uparrow x \lambda \uparrow y$ in $\beta_{2}(Q)$. Then, in particular, $\uparrow x \| \uparrow y$. Clearly, $u \leq v$ in $Q$ iff $\uparrow u \supseteq \uparrow v$ iff $\uparrow u \leq^{d} \uparrow v$ in $\left\langle F_{\text {hco }}(Q) ; \leq^{d}\right\rangle$. In particular, $u \| v$ in $Q$ iff $\uparrow u \| \uparrow v$ in $\left\langle F_{\text {hco }}(Q) ; \leq^{d}\right\rangle$. This yields $x \| y$. Hence $x \lambda y$ or $y \lambda x$ in $Q$. Since $y \lambda x$ would give a contradiction by the "only if" part, we obtain $x \lambda y$.

Lemma 3.22. For a quasiplanar diagram $Q, \beta_{1}(Q)$ makes sense, it is uniquely defined, it is a planar diagram of a slim semimodular lattice, and the diagrams $\beta_{1}(Q)$ and $\beta_{2}(Q)$ are the same, up to similarity. Furthermore, $\pi$ from Lemma 3.2 is a similarity map.

Proof. If $\beta_{1}(Q)$ exists, its uniqueness is evident from Definition 2.9. First, as a preparation to use Lemma 3.18, we show that if $X \in F_{\text {hco }}(Q)$, then

$$
X^{\text {lus }}=\uparrow \operatorname{lbe}(X) \text { and } X^{\text {rus }}=\uparrow \operatorname{rbe}(X) \text {. }
$$

It follows from Lemmas 3.1 and 3.2 that $X=\uparrow \operatorname{Min} \operatorname{Betw}(\operatorname{lbe}(X), \operatorname{rbe}(X))$. We know from Lemma 3.19 that the meet-irreducible elements of $\beta_{2}(Q)$ are exactly the $\uparrow x$ where $x \in Q \backslash\{0,1\}$. We have to consider the minimal ones above $X$, with respect to " $\leq d "$. That is, the maximal ones below $X$, with respect to set inclusion. Clearly, they are the members of $A=\{\uparrow x: x \in \operatorname{Min} \operatorname{Betw}(\operatorname{lbe}(X), \operatorname{rbe}(X))\}$. By definition, $X^{\text {lus }}$ is the leftmost member of $A$ with respect to $\lambda$ defined in $\beta_{2}(Q)$. Hence, by Lemma $3.21, X^{\text {lus }}=\uparrow \operatorname{lbe}(X)$. The rest of (3.16) follows similarly.

Next, consider the order-isomorphism $\pi:\left\langle F_{\text {hco }}(Q) ; \leq^{d}\right\rangle \rightarrow\langle E(Q) ; \leq\rangle$, defined by $X \mapsto\langle\operatorname{lbe}(X), \operatorname{rbe}(X)\rangle$ in Lemma 3.2. By Lemma 3.20, $\beta_{2}(Q)$ exists, it is uniquely defined, and it is a planar diagram of $\left\langle F_{\text {hco }}(Q) ; \leq^{d}\right\rangle$. Hence, $\langle E(Q) ; \leq\rangle$ has a unique diagram $D^{\prime}$ such that $\pi: \beta_{2}(Q) \rightarrow D^{\prime}$ is a similarity map. We know that $\pi$ is an order isomorphism. Therefore, it suffices to show that $D^{\prime}$ is a diagram of $\langle E(Q) ; \leq\rangle$ that satisfies $(2.2)$. Let $X_{1}, X_{2} \in F_{\text {hco }}(Q)$, and let $\left\langle x_{i}, y_{i}\right\rangle=\pi\left(X_{i}\right)=$ $\left\langle\operatorname{lbe}\left(X_{i}\right), \operatorname{rbe}\left(X_{i}\right)\right\rangle$, for $i \in\{1,2\}$. According to $(2.2)$, the concrete task is to show that

$$
X_{1} \lambda X_{2} \text { in } \beta_{2}(Q) \Longleftrightarrow x_{1} \lambda^{<} x_{2} \text { and } y_{1} \lambda^{>} y_{2} \text { in } Q \text {. }
$$

To prove the implication " $\Rightarrow$ ", assume $X_{1} \lambda X_{2}$. By Lemma 3.2, we have $X_{i}=\uparrow^{\text {hco }}\left\{x_{i}, y_{i}\right\}$ for $i \in\{1,2\}$. Using the notation introduced in Definition 2.10 
and arguing similarly as in the previous proof, we claim that

$$
\operatorname{lsp}\left(X_{i}\right)=F_{n_{i}} \Longleftrightarrow n_{i}=\min \left\{k: y_{i} \in F_{k}\right\}
$$

To see this, we can argue as follows: $\operatorname{lsp}\left(X_{i}\right)=F_{k} \Longleftrightarrow F_{k} \leq^{d} X_{i}$ and $F_{k}$ is maximal with respect to $\leq^{d} \Longleftrightarrow F_{k} \supseteq X_{i}$ and $F_{k}$ is minimal with respect to set inclusion $\Longleftrightarrow y_{i} \in F_{k}$ and $k$ is minimal; in the last step we used that $F_{k}$ is left-closed by Lemma 3.6 and $x_{i} \lambda^{=} y_{i}$, and so $y_{i} \in F_{k}$ implies $x_{i} \in F_{k}$. This proves (3.18).

From Lemma 3.15, we obtain $\operatorname{lsp}\left(X_{1}\right)>^{d} \operatorname{lsp}\left(X_{2}\right)$. This and (3.18) yield that $F_{n_{1}}:=\operatorname{lsp}\left(X_{1}\right) \subset \operatorname{lsp}\left(X_{2}\right)=: F_{n_{2}}, y_{1} \in F_{n_{1}}, y_{2} \in F_{n_{2}}$, and $y_{2} \notin F_{n_{1}}$ since we have $n_{1}<n_{2}$ by $F_{n_{1}} \subset F_{n_{2}}$. Since $y_{2} \notin F_{1}$, we have $y_{1} \neq y_{2}$. Hence, either $y_{1} \lambda^{>} y_{2}$, or $y_{2} \lambda^{>} y_{1}$. However, if $y_{2} \lambda^{>} y_{1}$, then $y_{2}$ belongs to $F_{n_{1}}$ since $y_{1} \in F_{n_{1}}$ and $F_{n_{1}}$ is a left-closed order filter by Lemma 3.6, and this is a contradiction. Consequently, $y_{1} \lambda^{>} y_{2}$. The left-right dual of the argument above gives $x_{1} \lambda^{<} x_{2}$. This proves " $\Rightarrow$ " in (3.17).

Finally, to prove the converse implication, assume that $x_{1} \lambda^{<} x_{2}$ and $y_{1} \lambda^{>} y_{2}$ hold in $Q$. If $X_{1} \leq X_{2}$, then (2.1) yields $x_{1} \lambda \leq x_{2}$ and $y_{2} \lambda^{\geq} y_{1}$ since $\pi$ is an orderisomorphism, and this contradicts $y_{1} \lambda^{>} y_{2}$. We obtain similarly that $X_{2} \leq X_{1}$ contradicts $x_{1} \lambda^{<} x_{2}$. Therefore, $X_{1} \| X_{2}$. If $X_{2} \lambda X_{1}$, then the $\Rightarrow$ direction of (3.18) yields $x_{2} \lambda^{<} x_{1}$, which contradicts $x_{1} \lambda^{<} x_{2}$. The only remaining possibility is $X_{1} \lambda X_{2}$. This proves the implication " $\Leftarrow$ " in (3.17).

3.5. The end of the proof. Armed with the auxiliary statements presented so far, now we are in the position to accomplish our goal.

Proof of Theorem 2.11. By Lemma 3.22, $\beta_{1}(Q)$ equals $\beta_{2}(Q)$, up to similarity. Hence, in each part of the proof, it suffices to deal with one of $\beta_{1}$ and $\beta_{2}$.

Part (i) is Lemma 3.9, while Part (ii) follows from Lemmas 3.5, 3.6, and 3.13.

To prove Part (iii), let $D$ be a finite planar slim semimodular lattice diagram, and let $Q=\alpha(D)$. Define a map $\kappa: D \rightarrow \beta_{1}(Q)$ by $x \mapsto\left\langle x^{\text {lus }}, x^{\text {rus }}\right\rangle \in E(Q)$. (Here, for technical reasons, we extend the definition of $x^{\text {lus }}$ and $x^{\text {rus }}$ by letting $1^{\text {lus }}=1^{\text {rus }}=1$; this will cause no problem and makes the definition of $\kappa$ meaningful.) Since $x=$ $x^{\text {lus }} \wedge x^{\text {rus }}$ by Lemma 3.17, $\kappa$ is injective. Assume $\langle y, z\rangle \in E(Q)$ such that $y \neq z$, and define $x$ by $x=y \wedge z$. This is an irredundant meet representation since $y \| z$. By the uniqueness part of Lemma 3.17 and $y \lambda z$, we obtain $\langle y, z\rangle=\left\langle x^{\text {lus }}, x^{\text {rus }}\right\rangle=\kappa(x)$. Also, if $\langle x, x\rangle \in E(Q)$, then $\kappa(x)=\left\langle x^{\text {lus }}, x^{\text {rus }}\right\rangle=\langle x, x\rangle$. Hence, $\kappa$ is surjective. Finally, comparing Lemma 3.18(i) to (2.1) and Lemma 3.18(ii) to (2.2), we conclude that $\kappa$ is similarity map. This proves Part (iii).

To prove Part (iv), let $Q$ be a quasiplanar diagram. Combining Lemmas 3.19 and 3.22, we conclude $\operatorname{Mi}\left(\beta_{1}(Q)\right)=\{\langle x, x\rangle: x \in Q \backslash\{0,1\}\}$. To form $\left.\alpha\left(\beta_{1}(Q)\right)\right)$, we have to add a bottom and a top to $\operatorname{Mi}\left(\beta_{1}(Q)\right)$; denote them by $\langle 0,0\rangle$ and $\langle 1,1\rangle$, respectively. Then we have $\alpha\left(\beta_{1}(Q)\right)=\{\langle x, x\rangle: x \in Q\}$. We claim that $\gamma: Q \rightarrow \alpha\left(\beta_{1}(Q)\right)$, defined by $x \mapsto\langle x, x\rangle$, is a similarity map. Obviously, $\gamma$ is a bijection. Since the position of a top or bottom element in a diagram is unique up to similarity, it suffices to deal with the elements of $Q \backslash\{0,1\}$. Assume $x, y \in Q \backslash\{0,1\}$. Based on (2.1), we have

$$
\langle x, x\rangle \leq\langle y, y\rangle \Longleftrightarrow x \lambda^{\leq} y \text { and } y \lambda^{\geq} x \Longleftrightarrow x \leq y
$$


which shows that $\gamma$ is an order-isomorphism. Based on (2.2), we obtain

$$
\langle x, x\rangle \lambda\langle y, y\rangle \Longleftrightarrow x \lambda^{<} y \text { and } x \lambda^{>} y \Longleftrightarrow x \lambda y .
$$

Therefore, $\gamma$ is a similarity map, completing the proof of Part (iv).

Proof of Corollary 2.12. Besides Theorem 2.11, the proof is based on two facts. First, let $X(k)$ denote the set of planar slim semimodular lattice diagrams of length $k$, understood up to similarity. We know from Czédli and Schmidt [19], see also Czédli and Grätzer [15], that there exists a bijection between $X(k)$ and the set $S_{k}$ of permutations acting on $\{1, \ldots, k\}$. Second, it follows easily from Dilworth [21] or Adaricheva, Gorbunov and Tumanov [4, Theorem 1.7.(1-2)] and, furthermore, it is explicitly stated in Czédli and Schmidt [19, Corollary 2.4] that for every slim semimodular lattice $K,|\mathrm{Mi} K|=\operatorname{length}(K)$. So if $D$ is a planar semimodular lattice diagram, then $|\mathrm{Mi} D|=$ length $(D)$. Taking Definition 2.8 into account, we obtain that $|Q|=|\alpha(D)|=2+|\operatorname{Mi} D|=2+\operatorname{length}(D)$. Therefore, Theorem 2.11 gives a bijective correspondence between the set of $n$-element quasiplanar diagrams, understood up to similarity, and $X(n-2)$. Thus, the number of $n$-element quasiplanar diagrams, understood up to similarity, is $|X(n-2)|=\left|S_{n-2}\right|=(n-2)$ !.

\section{Comments And examples}

One may ask which finite bounded posets have quasiplanar diagrams.

Proposition 4.1. A finite bounded partially ordered set $P$ has a quasiplanar diagram iff its order dimension is at most two.

Proof. Assume that $P$ has a quasiplanar diagram. By Theorem 2.11, $P$ can be order-embedded into a finite slim semimodular lattice $L$. Since $L$ has a planar diagram by Lemma 3.13, cited from Czédli and Schmidt [17], it is of order-dimension at most two. Hence, $L$ has an order embedding into the direct product of two chains; see the paragraph after Lemma 3.7. Thus, $P$ also has an embedding into this direct product, and it is of order-dimension at most two.

Next, assume that $P$ is of order-dimension at most two. Then $P$ has a diagram $Q$ that is a subdiagram of a grid $G$. By (3.10), $Q$ is a quasiplanar diagram.

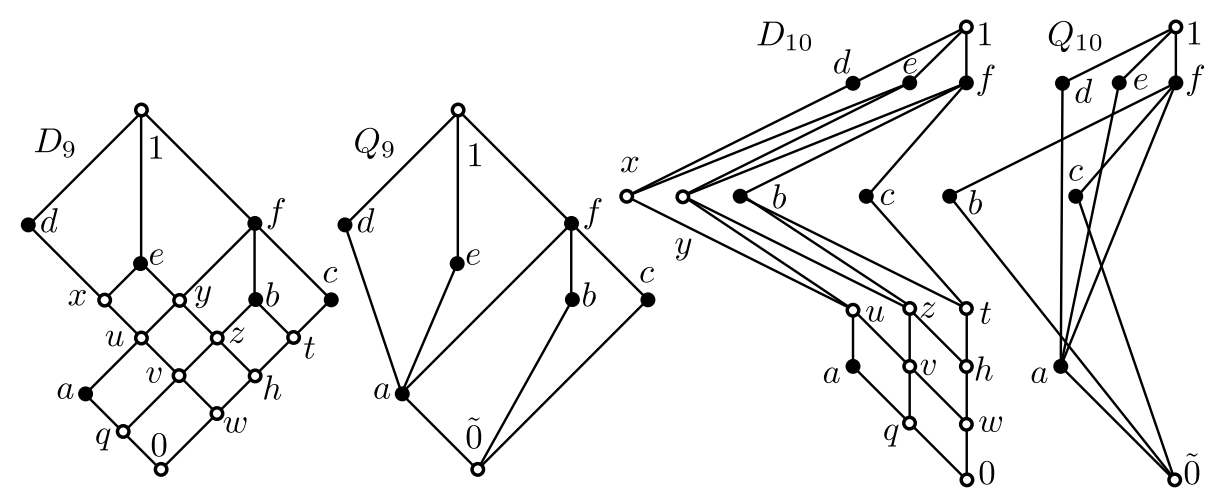

Figure 7. $D_{9}=D_{10}$ and $Q_{9}=\alpha\left(D_{9}\right)$, but $Q_{10} \neq \alpha\left(D_{10}\right)$

We conclude the paper with some additional examples and comments. Figure 3, where $Q_{i}=\alpha\left(D_{i}\right)$ for $i \in\{4, \ldots, 7\}$, explains why we deal with diagrams rather 
than lattices and posets: $Q_{4}$ and $Q_{5}$ show that order-isomorphic quasiplanar diagrams can determine non-isomorphic lattices. Also, $D_{6}$ is the smallest planar slim semimodular lattice diagram such that $Q_{6}=\alpha\left(D_{6}\right)$ is not planar, and there is no planar diagram order-isomorphic to $\alpha\left(D_{6}\right)$. Figure 7 illustrates that Lemma 3.9 is not as obvious as it may look. In the figure, $D_{9}$ and $D_{10}$ are equal, up to similarity. For $i \in\{9,10\}, Q_{i}$ is obtained from $D_{i}$ by omitting vertices and connecting the remaining ones, without changing their position in the plane. We have $Q_{9}=\alpha\left(D_{9}\right)=\alpha\left(D_{10}\right)$. However, $Q_{10} \neq \alpha\left(D_{10}\right)$, because $Q_{10}$ is not a quasiplanar diagram since $c \| a, c$ is on the left of the chain $\{0, a, f, 1\}$ through $a$, but $c$ is on the right of the chain $\{0, a, d, 1\}$ through $a$. Figure 7 explains the parenthesized comment in Definition 2.8(i). Finally, Figure 1 indicates how $\alpha$ acts in case of vertical decompositions (into so-called glued sums), provided the bottom element of the upper lattice diagram, $D_{2}$, is meet-reducible. The idea suggested by the figure was used in an earlier proof of Lemma 3.9.

Acknowledgment. The author is indebted to both referees for careful reading and useful hints. The first version of the paper claimed, in essence, that the implication

$$
(\mathrm{A} 1) \Rightarrow((\mathrm{A} 2) \text { and }(\mathrm{A} 3))
$$

"is an obvious extension of Proposition 1.7 in Kelly and Rival [27]". The author is particularly grateful to the anonymous referee who pointed out that this implication is false.

\section{REFERENCES}

[1] Abels, H.: The geometry of the chamber system of a semimodular lattice. Order 8, 143-158 (1991)

[2] Adaricheva, K.: Representing finite convex geometries by relatively convex sets. European Journal of Combinatorics 37, 68-78 (2014)

[3] Adaricheva, K., Czédli, G.: Notes on the description of join-distributive lattices by permutations. Algebra Universalis 72, 155-162 (2014)

[4] Adaricheva, K., Gorbunov, V.A., Tumanov, V.I.: Join-semidistributive lattices and convex geometries. Advances in Math. 173, 1-49 (2003)

[5] Armstrong, D.: The Sorting Order on a Coxeter Group. Journal of Combinatorial Theory Series A 116, 1285-1305 (2009)

[6] Avann, S.P.: Application of the join-irreducible excess function to semimodular lattices. Math. Annalen 142, 345-354 (1961)

[7] Caspard, N., Monjardet, B.: Some lattices of closure systems on a finite set. Discrete Mathematics and Theoretical Computer Science 6, 163-190 (2004)

[8] Czédli, G.: The matrix of a slim semimodular lattice. Order 29, 85-103 (2012)

[9] Czédli, G.: Representing homomorphisms of distributive lattices as restrictions of congruences of rectangular lattices. Algebra Universalis 67, 313-345 (2012)

[10] Czédli, G.: Coordinatization of join-distributive lattices. Algebra Universalis 71, 385-404 (2014)

[11] Czédli, G.: Finite convex geometries of circles. Discrete Mathematics 330, 61-75 (2014)

[12] Czédli, G.: The asymptotic number of planar, slim, semimodular lattice diagrams. Order, to appear; DOI 10.1007/s11083-015-9361-0; http://arxiv.org/abs/1206.3679

[13] Czédli, G., Dékány, T., Ozsvárt, L., Szakács, N., Udvari, B.: On the number of slim, semimodular lattices. Mathematica Slovaca, to appear; http://arxiv.org/abs/1208.6173

[14] Czédli, G., Grätzer, G.: Notes on planar semimodular lattices. VII. Resections of planar semimodular lattices. Order 30, 847-858 (2013)

[15] Czédli, G., Grätzer, G.: Planar semimodular lattices and their diagrams. Chapter 3 in: Grätzer, G., Wehrung, F. (eds.) Lattice Theory: Special Topics and Applications. Birkhäuser Verlag, Basel (2014) 
[16] Czédli, G., Ozsvárt, L., Udvari, B.: How many ways can two composition series intersect?. Discrete Mathematics 312, 3523-3536 (2012)

[17] Czédli, G., Schmidt, E.T.: The Jordan-Hölder theorem with uniqueness for groups and semimodular lattices. Algebra Universalis 66, 69-79 (2011)

[18] Czédli, G., Schmidt, E.T.: Slim semimodular lattices. I. A visual approach. Order 29, 481-497 (2012)

[19] Czédli, G., Schmidt, E.T.: Composition series in groups and the structure of slim semimodular lattices. Acta Sci Math. (Szeged) 79, 369-390 (2013)

[20] Czédli, G., Schmidt, E.T.: Slim semimodular lattices. II. A description by patchwork systems. Order 30, 689-721 (2013)

[21] Dilworth, R.P.: Lattices with unique irreducible decompositions. Annals of Mathematics (2) 41, 771-777 (1940)

[22] Grätzer, G.: Lattice Theory: Foundation. Birkhäuser Verlag, Basel (2011)

[23] Grätzer, G., Knapp, E.: Notes on planar semimodular lattices. I. Construction. Acta Sci. Math. (Szeged) 73, 445-462 (2007)

[24] Grätzer, G., Knapp, E.: Notes on planar semimodular lattices. III. Congruences of rectangular lattices. Acta Sci. Math. (Szeged), 75, 29-48 (2009)

[25] Grätzer, G., Knapp, E.: Notes on planar semimodular lattices. IV. The size of a minimal congruence lattice representation with rectangular lattices. Acta Sci. Math. (Szeged), 76, 3-26 (2010)

[26] Jamison-Waldner, R.E.: Copoints in antimatroids. In: Combinatorics, graph theory and computing, Proc. 11th southeast. Conf., Boca Raton/Florida 1980, Vol. II, Congr. Numerantium 29, 535-544 (1980)

[27] Kelly, D., Rival, I.: Planar lattices. Canad. J. Math. 27, 636-665 (1975)

[28] Monjardet, B.: A use for frequently rediscovering a concept. Order 1, 415-417 (1985)

[29] Nation, J. B.: Notes on Lattice Theory. http://www.math.hawaii.edu/ jb/books.html

[30] Schmidt, E.T.: Congruence lattices and cover preserving embeddings of finite length semimodular lattices. Acta Sci. Math. Szeged 77, 47-52 (2011)

[31] Stern, M.: Semimodular Lattices. Theory and Applications, Encyclopedia of Mathematics and its Applications 73. Cambridge University Press (1999)

[32] Ward, M.: Structure Residuation. Annals of Mathematics (2) 39, 558-568 (1938)

E-mail address: czedli@math.u-szeged.hu

URL: http://www.math.u-szeged.hu/ czedli/

University of Szeged, Bolyai Institute. Szeged, Aradi vértanúk tere 1, Hungary 6720 